\title{
Übergemeindliche Verbindungen im Urchristentum nach dem Galaterbrief
}

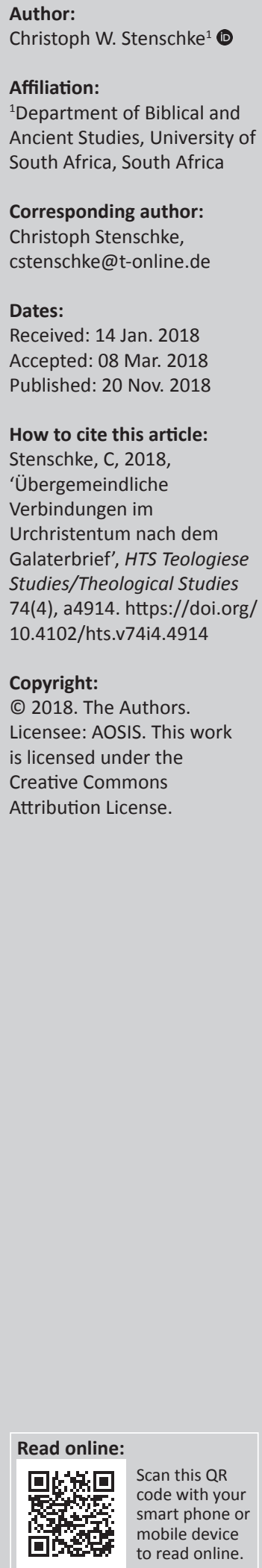

While many studies of early Christianity mention translocal links as one of the significant characteristics of early Christian communities, these links have not been examined in detail. The present article provides a brief survey of research and discusses the significance of such links. It then examines in detail the evidence for such links in Paul's Letter to the Galatians. The article is the first examination of the direct and indirect translocal links in Galatians and of their function in the letter. A final section draws out the implications of understanding early Christianity.

\section{Einführung}

Obwohl wiederholt und in ganz unterschiedlichen Zusammenhängen angeführt, ${ }^{1}$ sind die überörtlichen Beziehungen urchristlicher Gemeinden bisher noch wenig systematisch untersucht worden. $\mathrm{Zu}$ ihrer Bedeutung sind einige Aspekte einführend knapp zu erwähnen. In meinen Aufsätzen zu übergemeindlichen Verbindungen in der Apostelgeschichte, im Römerbrief und dem 1. und 2. Korintherbrief habe ich einleitend auf die Bedeutung übergemeindlicher Verbindungen für die Frage nach der Vergleichbarkeit von neutestamentlichen Gemeinden und antiken Vereinen hingewiesen (Stenschke 2011, 2017). Während übergemeindliche Verbindungen für viele Forscher einen wichtigen Unterschied zwischen Gemeinden und Vereinen konstituieren, stellen andere diesen Konsens in Frage. Sie argumentieren zum einen für überörtliche Verbindungen auch zwischen antiken Vereinen und zum anderen hinterfragen sie Ausmaß und Bedeutung übergemeindlicher Verbindungen im Urchristentum. Die vorhandenen Verbindungen seien im Wesentlichen auf die Person des Paulus beschränkt, so dass man daher kaum von übergemeindlichen Verbindungen sprechen könne. Angesichts dieser Forschungslage ist es notwendig, die überörtlichen Verbindungen zwischen urchristlichen Gemeinden neu und umfassend zu untersuchen um festzustellen, ob und inwieweit sich überörtliche Verbindungen tatsächlich aufzeigen lassen. Dabei verdient die Rolle des Paulus und seiner Mitarbeiter und Missionspartner besondere Aufmerksamkeit. ${ }^{2}$ Sind Paulus und seine Mitarbeiter tatsächlich die primäre, wenn nicht einzige, überörtliche Verbindung zwischen verschiedenen urchristlichen Gemeinden? ${ }^{3}$

Neben dieser Vergleichbarkeit spielen übergemeindliche Verbindungen eine bisher vernachlässigte Rolle in der Rekonstruktion der Geschichte des Urchristentums sowie bei Einleitungsfragen zum Neuen Testament. Ferner wäre zu fragen, welche Bedeutung sie in der Diskussion der theologischen Einheit und Vielfalt im Neuen Testament haben. Im Einzelnen ist auch in der gegenwärtigen Betonung der Rhetorik in der Paulus-Forschung ('rhetorical criticism') nach der Funktion der direkt oder indirekt erscheinenden übergemeindlichen Verbindungen in der Argumentation des Paulus zu fragen. Die übergemeindlichen Verbindungen sind ferner von Bedeutung für die Anwendung der Methoden der sozialen Netzwerkanalyse auf antike Verbindungen, vor allem das Netzwerk paulinischer Gemeinden. Trotz Vorarbeiten und Einzelstudien liegt keine größere Studie zu diesem Thema vor.

1.So etwa auch von Hezser (2011). Sie betont die Mobilität des Ürchristentums, die einen wesentlichen Anteil an der materiellen Grundlage übergemeindlicher Beziehungen bildet: 'Ancient Christians are also considered to have been very mobile, as wandering charismatics in Roman Palestine and the Eastern Mediterranean and as missionaries in various parts of the Roman empire, and this mobility is believed to have been an important factor in the dissemination and eventual 'triumph' of Christianity' (S. 1).

2.Detaillierte Auseinandersetzung und Kritik an Ascough (1997) bei Schmeller (2001:130-133). Schmeller hinterfragt Ascoughs Hinweise auf den überregionalen Charakter antiker Vereine (S. 131-132) und verweist knapp auf Hinweise auf übergemeindliche Verbindungen. Schmeller schließt: 'Alles in allem hat Ascough zwar nachgewiesen, dass es in begrenztem Umfang Vereine mit translokalen Aspekten gab; seine These einer Gleichartigkeit von Vereinen und Gemeinden in dieser Hinsicht hat er jedoch nicht bewiesen, ja nicht einmal gab; seine These einer Gleichartigke
wahrscheinlich gemacht' (S. 133).

3.Nach Schmeller (2001) war: 'Diese überregionale Gemeinschaft [...] nicht an die Person des Paulus gebunden. Gegen Ende des 1. Jhdt. n. Chr. wurden die Paulusbriefe gesammelt. Die Pastoralbriefe setzen als Adressaten Gemeinden in einem größeren Gebiet voraus. Vernetzt waren aber offenbar auch die johanneischen Gemeinden, wie 1-3 Joh zeigen' (S. 133). 
Auf diesem Hintergrund sollen die im Galaterbrief direkt und indirekt erwähnten und im Kontext seiner Entstehung vorausgesetzten überörtlichen Verbindungen so vollständig wie möglich erfasst werden. An einigen Stellen erscheinen sie zweifelsfrei und direkt, an anderen Stellen nur indirekt. An manchen Stellen bleibt es unklar, ob und welche übergemeindlichen Verbindungen tatsächlich impliziert sind. Das Maß an Gewissheit wird jeweils differenziert angegeben. Obwohl bei der Zusammenfassung und Analyse alle möglichen Vorkommen berücksichtigt werden, liegt das Gewicht bei den direkten Erwähnungen.

Der Umfang tatsächlicher übergemeindlicher Verbindungen hängt entscheidend davon $\mathrm{ab}$, ob und wie man zwischen persönlichen Verbindungen des Paulus zu einzelnen Leuten in anderen Gemeinden und direkten übergemeindlichen Verbindungen differenzieren kann und muss. Wo ist tatsächlich von Verbindungen von Gemeinden qua Gemeinden auszugehen, wo geht es - strenggenommen nur um Verbindungen zwischen Einzelpersonen? Würde man Paulus und andere erwähnte Personen als reine Privatpersonen verstehen, blieben nur wenige Stellen, die explizit von Verbindungen zwischen Gemeinden auf beiden oder mehreren Seiten berichten. ${ }^{4}$ Gegen diese Einschränkung spricht, dass Paulus und die namentlich genannten Personen in Gemeinden eingebettet sind: sie kommen aus Gemeinden, wurden von Gemeinden ausgesandt, gründen Gemeinden, wirken in Gemeinden, repräsentieren Gemeinden und grüßen Gemeinden beziehungsweise übermitteln Grüße von Gemeinden. ${ }^{5}$ Schnelle (2014, kursiv CS) beobachtet:

Die Mehrheit der in den Paulusbriefen erwähnten Mitarbeiter waren Gemeindegesandte. Sie entstammten den von Paulus gegründeten Gemeinden und nahmen nun als Delegierte dieser Gemeinden an der Missionsarbeit teil [...] Sie hielten den Kontakt zu ihren Heimatgemeinden aufrecht, unterstützten Paulus auf vielfältige Weise und missionierten eigenständig im Umland der paulinischen Gemeindegründungen. (S. 143)

Von daher entstehen durch sie in der Tat übergemeindliche Verbindungen. So schließt Y.-H. Park (2015):

Letter writing in itself was an activity of a translocal nature. Paul

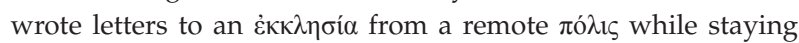

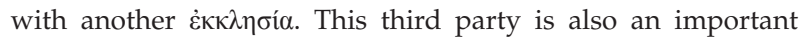
factor in the epistolary dynamics. Paul placed these congregations in his greetings, parallel to his name. (S. 112)

Obwohl er darin gewiss eine zentrale Stellung einnimmt, entsteht die Gemeinschaft der Gemeinden nicht erst durch Paulus.

\footnotetext{
4.Dabei wäre zu fragen, wie solche Kontakte von ganzen Gemeinden zu ganzen Gemeinden unter antiken Bedingungen ausgesehen haben könnten. Ein instruktive frühchristliches Beispiel wäre der 1. Clemensbrief, in dem angeblich 'Die Kirche Gottes, die Rom [...] bewohnt, an die Kirche Gottes, die Korinth als Fremde bewohnt' (1:1) schreibt. Freilich handelt es sich auch dabei nicht um das Gemeinschaftswerk einer ganzen Gemeinde.

5.Während der Abfassung des Galaterbriefs war Paulus an anderen Orten in Gemeinden aktiv. Vergleiche dazu Thompson (1998:53-54). Thompson sieht nicht Paulus, sondern Gemeinden an entscheidenden Stellen im 'heiligen Netzwerk'. 'The Paulus, sondern Gemeinden an entscheidenden Stellen im 'heiligen Netzwerk': 'The network 'servers of the holy internet were churches, where Christian traditions wer function. Created, remembered, shaped, and dispersed. [...] They effectively functioned as the junction or meeting point through which messages passed to and from individuals and other congregations' (S. 53). Zu Paulus und den verschiedenen Bezügen zu 'seinen' Gemeinden vergleiche auch Best (1988).
}

Paulus war mit der gegenwärtigen Situation der Galater vertraut und spricht sie im Brief direkt an. Schon diese Vertrautheit ist Indiz für übergemeindliche Verbindungen (wohl zwischen einzelnen Mitgliedern oder Gruppen innerhalb der Gemeinde und dem Apostel), ohne die Paulus nicht entsprechend informiert gewesen wäre. Im Folgenden werden diese Hinweise ebenfalls aufgenommen. Wir folgen dem Aufbau des Briefs, fassen aber inhaltlich zusammengehörende Aussagen an der ersten Stelle ihres Vorkommens zusammen. Nach einer Zusammenfassung zeigen wir abschließend die Bedeutung der vorhandenen Hinweise auf übergemeindliche Verbindungen für die einführend benannten fünf Themen.

\section{Übergemeindliche Verbindungen im Galaterbrief}

\section{Galater 1}

Am Anfang seines Briefes erwähnt Paulus mehrere Männer aus seinem Umfeld: '[U]und alle Brüder, die bei mir sind' (Gal 1:2). Auch wenn er sie von sich und seinem besonderen Amt unterscheidet, macht Paulus deutlich, dass er in Gemeinschaft arbeitet und schreibt. Er steht keineswegs isoliert da. Bei seinem Anliegen und Brief handelt es sich nicht um eine Privatangelegenheit. Zur Funktion der Erwähnung 'aller Brüder' schreibt Moo (2013:70): Paulus 'undoubtedly includes this wider group to lend strength to his appeal: the views he is teaching in the letter are not his alone but are widely held. ${ }^{\prime}$ Bei der Erwähnung sämtlicher Brüder bei ihm geht es weniger um seine Autorität:

sondern $[u m]$ einerseits die völlige Isolation, in die sich die Galater verrannt haben, andererseits den einmütigen Konsens, in dem er, Paulus, steht und dem seine Ausführungen entspringen. Alle stehen sie hinter ihm. Das heißt, nimmt man Paulus beim Wort und verdächtigt ihn nicht der Übertreibung, nichts anderes, als dass die Lage in Galatien im Kreise der um Paulus versammelten Brüder erörtert, die Entgegnung gemeinsam besprochen und von allen gutgeheißen wurde. Allen voran antwortet Paulus, aber neben ihm verantworten 'alle Brüder' den Inhalt des Schreibens mit und verleihen ihm Nachdruck. [...] Was alle [...] bedenken und gutheißen, bildet die Grundlage für die Entgegnung des Paulus, die er zum Diktat formuliert (Gal 6:11). [...] So trägt das Schreiben zwar die Handschrift des Paulus, darf jedoch auch nicht einfach nur als seine eigene Ausführung verstanden werden. Vielmehr ist die Diskussion mit den 'Brüdern' in sie eingeflossen. (S. 185-186) ${ }^{7}$

Auch diese Männer sind mit den Gemeinden Galatiens verbunden und dürften - bei einer Spätdatierung des Briefs selbst als Abgesandte und Repräsentanten von Gemeinden

6.Die Identifikation der Brüder hängt von der Datierung des Briefs ab. Bei einer Frühdatierung (Südgalatien bzw. Provinzhypothese) wäre an die Leiter de antiochenischen Gemeinde inklusive Barnabas zu denken, bei einer späteren antiochenischen Gemeinde inklusive Barnabas zu denken, bei einer spateren Missionsreise beziehungsweise Gemeindemitglieder am Ort der Abfassung.

7.Ollrog (1979:185-186); vergleiche auch Öhler (2013); zur Beteiligung bei der Verfassung von Briefen vergleiche S. 255-256. Die Erwähnung anderer Absender ist 'ein deutlicher Hinweis darauf, dass er [Paulus] die Briefe [...] an Absender ist 'ein deutlicher Hinweis darauf, dass er [Paulus] die Briefe [...] an
die Gemeinden im Kontext seines Teams verfasste und auch als Mitteilungen der Verkündigungsgemeinschaft verstanden wissen wollte' (S. 256). 
bei Paulus sein. Über ihre genauen Tätigkeiten sind keine Details bekannt. $^{8}$

Paulus schreibt 'an die Gemeinden in Galatien' einen Rundbrief. Dessen Verbreitung über mehrere Gemeinden hinweg setzt entsprechende überörtliche Verbindungen voraus oder stiftet sie. Eventuell wurde der Brief, nach Erhalt durch einen Boten von Paulus, von den Gemeinden beziehungsweise den von ihnen beauftragten Boten selbst weitergereicht (d.h., sie wussten vielleicht nicht von allen Gemeinden in Galatien, aber doch von Gemeinden in ihrer Nähe). ${ }^{9}$ Alternativ könnte ein Bote oder Boten des Paulus mit der Verlesung des Briefs in mehreren Gemeinden betraut worden sein, weil Paulus sich nicht auf die Weitergabe des Briefs im anvisierten Gebiet verlassen wollte oder konnte. Davon geht Bruce (1982:72) aus:

It was evidently a circular letter, designed to be taken by a messenger to one of the Galatian churches, then to the next on his itinerary, and so on until each church had heard its contents. [...] Paul apparently did not send several copies, one for each church; his words in 6:11 imply that each church would see the one copy that he sent and take note of the 'large letters' that characterized his own handwriting. (S. 254-255) $)^{10}$

Im ersten Fall entstehen übergemeindliche Verbindungen durch die (unterschiedlichen) Boten der Gemeinden, im zweiten Fall durch den oder die Boten des Paulus.

Von außen waren Irrlehrer in die Gemeinden Galatiens eingedrungen ('nur dass einige da sind, die euch verwirren', Gal 1:7). ${ }^{11}$ Da Paulus von ihnen in der 3. Person spricht, während er die Menschen, die durch seinen Dienst zum Glauben gekommen waren, in der 2. Person spricht, ist es unwahrscheinlich, dass die Gegner aus den Gemeinden selbst stammten (vgl. Bruce 1982:82). Durch sie entstehen ebenfalls übergemeindliche Verbindungen, auch wenn sie nicht im Sinn des Paulus waren. Die Irrlehrer waren wahrscheinlich ebenso wie Paulus und die eingangs erwähnten Brüder in Gemeinden verankert, wirkten in verschiedenen Gemeinden und waren, je nach Rekonstruktion, eventuell in deren Auftrag unterwegs.

Von den Gegnern ist nochmal knapp in Galater 4:17 die Rede. Sie wollen die Galater als ihre Anhänger werben, damit die Galater wiederum um sie werben. ${ }^{12}$ Ihr Ziel ist es, die Galater von Paulus abspenstig $\mathrm{zu}$ machen und damit von der Gemeinschaft mit dem Christus, dessen Apostel Paulus ist 8.Vergleiche den überblick bei Öhler (2013:253-256) und Dickson (2003:86-132).

9.Dies scheint die in Koloser 4:16 anvisierte Vorgehensweise zu sein, vergleiche dazu E. Schweizer (1989:179).

10.Zur Bedeutung der Mitarbeiter des Paulus als Gesandte und Boten vergleiche Öhler (2013:254-255). Die Fragestellung gilt in Analogie für den Epheserbrief verstanden als Rundbrief beziehungsweise für die Johannesoffenbarung und ihre Sendschreiben an mehrere Gemeinden.

11.Vergleiche zu Anlass und Zweck des Briefs den Überblick bei Moo (2013:19-31) Zur Identität und Herkunft der Gegner vergleiche Sumney (1999:79-133); vergleiche auch Sumney (2005) und Taylor (2005:115-122).

12.Bruce (1982:212) schreibt: 'Those people, says Paul to his converts, want you to count yourselves as exclusively their followers, enrolled members of their school.'
(Gal 5:4). Indirekt und in polemischer Abgrenzung erscheinen sie auch in Galater 5:7-8,10,12. ${ }^{13}$

In Galater 6:12 werden die Gegner konkreter charakterisiert als Menschen, die Ansehen haben wollen nach dem Fleisch und die Verfolgung um des Kreuzes Christi willen vermeiden wollen. ${ }^{14}$ Hier kann es nicht um eine detaillierte Rekonstruktion von deren Identität, Herkunft oder Verkündigung gehen. Wenn man Bruce und Jewett folgt, handelt es sich bei den 'Eindringlingen' um Judenchristen aus Jerusalem beziehungsweise Judäa. Sie suchen Verbindungen zu anderen Gemeinden (d.h., in Galatien und evtl. anderswo) und Einfluss auf die dortige Gemeindepraxis zu gewinnen (Forderung der Beschneidung von Heidenchristen und Gesetzesobservanz), um ihre eigene - zugegebener Weise - schwierige Situation zu Hause zum Besseren zu wenden: Bei Beschneidung und Gesetzesobservanz der Heidenchristen stünden die Judenchristen in Judäa in den schwierigen politischen Verhältnissen der 40er und 50er Jahre besser da: Sie hätten nun keinen höchst umstrittenen Kontakt mehr zu Heiden, sondern zu jüdischen Proselyten, und würden jüdische Identität durch eine 'übertrittsfreie' Aufnahme von Heiden in das Gottesvolk nicht länger relativieren oder kompromittieren. Dies setzt voraus, dass die Verhältnisse in Galatien in Jerusalem beziehungsweise Judäa vor und nach dem Wirken der Irrlehrer auch jenseits der christlichen Gemeinde bekannt waren oder zumindest bekannt werden konnten. Hier ist an jüdische Festpilger oder Gesandte zu denken. ${ }^{15}$ Diese Zielsetzung (durch Rückkopplung Veränderung und Verbesserung der eigenen Verhältnisse vor Ort) ist auch ein Motiv für übergemeindliche Verbindungen über weite Entfernungen hinweg. Neben diesen expliziten Vorkommen stehen die Irrlehrer und die von ihnen ausgehende Gefahr durchweg im Hintergrund der Argumentation des Paulus.

Aus diesen Hinweisen wird deutlich, dass andere Christen neben Paulus und seinen Mitarbeitern in den von Paulus und seinen Mitarbeitern gegründeten Gemeinden wirken und aus der Sicht des Paulus - Verwirrung stiften (vgl. auch Gal 2:12). Sie waren von außen in die von Paulus gegründeten Gemeinden gekommen. Dies setzt voraus, dass sie wussten, an welchen Orten es diese Gemeinden gab (vgl. der Bericht nach der ersten Missionsreise in Apg 14:26-28) und dass sie diese Gemeinden gezielt aufgesucht haben. ${ }^{16}$ Inwieweit

13.Paulus verwendet die rhetorische Strategie der 'severance; vergleiche Hansen (1993:824-825). Hansen schreibt: 'Paul's argument is designed to drive a wedge between the Galatian believers and the false teachers so as to split them apart' (S. 824)

14.Vergleiche Bruce (1982): 'This clause of purpose provides a strong argument in support of $R$. Jewett's thesis that Jewish Christians in Judea were stimulated by Zealotic pressure into a nomistic campaign among their fellow Christians in the late forties and early fifties' (S. 269); vergleiche Jewett (1970-1971:205). Zur politischen Situation in Judäa vergleiche Reicke (1982:200-226)

15.Vergleiche Hezser (2011:365-388). Die von ihr beschriebenen materiellen Grundlagen von antiken Reisen (S. 19-196) gilt auch für christliche Reisende.

16.Zu Mobilität und Kommunikation vergleiche Thompson (1998), 'Internet' Bauckham (1998). Bauckham (S. 25-35) verweist auf ausgeprägte Mobilität und Kommunikation im 1. Jahrhundert, auf den 'strong sense of itself as a worldwide movement' im Urchristentum (S. 27), auf die Mobilität urchristlicher Leiter und den movement' im Urchristentum 
durch diese Leute auch Verbindungen zu den Gemeinden beziehungsweise der Gemeinde entstanden sind, aus denen oder aus der sie kamen, ist nicht festzustellen. Ähnlich wie Paulus dürften auch sie auf einen (vermeintlichen oder echten) Konsens in einem größeren Kreis von Gemeinden verwiesen haben, um ihre Position zu stützen. Dass diese Leute - zum Leidwesen des Paulus - zumindest von einigen Galatern freundlich aufgenommen und mit ihrer Botschaft ernst genommen wurden, deutet an, dass reisende Missionare und Lehrer an sich nicht ungewöhnlich waren und gemeindeübergreifend Vertrauen genossen. Auch wenn Paulus in seinen Briefen, wie zu erwarten, den Mittelpunkt im Netzwerk übergemeindlicher Verbindungen bildet, entstehen übergemeindliche Verbindungen keineswegs ausschließlich durch ihn. ${ }^{17}$

Im Rahmen des autobiografischen Rückblicks ${ }^{18}$ schreibt Paulus, dass die Galater von seinem früheren Leben im Judentum gehört hätten (Gal 1:13). Schäfer (2004:78) schreibt: 'Die erste Frage, die sich unbefangen [...] stellt, lautet: Durch wen haben die galatischen Gemeinden von der früheren Verfolgungstätigkeit des Paulus erfahren?' In Betracht kommt zunächst Paulus selbst, der auch an anderen Stellen offen über seine frühere Tätigkeit spricht (vgl. 1 Kor 15:9; Phil 3:6). Doch nach Schäfer ist es plausibler:

$[D]$ ass nicht Erzählungen des Paulus als Quelle ihres Wissens fungierten, sondern sie von anderen über sein früheres Engagement gegen die Gemeinde informiert worden sind. Dieses Verständnis scheint besser zur distanzierten Formulierung in Galater 1:13 zu passen. (Schäfer 2004:79) ${ }^{19}$

Dies entspricht auch einer Angabe im weiteren Zusammenhang: Galater 1:22-23 setzt voraus, dass von der Lebenswende des Paulus inklusive seiner früheren Tätigkeit als Verfolger erzählt wurde. Auch wenn diese Bemerkung explizit nur den judäischen Raum erwähnt, in dem dies geschehen sein soll, setzt sie nach Schäfer (2004:150-152) ähnliches für den syrisch-kilikischen Raum voraus. Sie schreibt:

Wie sich dieses Wissen bis hinein in den kleinasiatischen Raum weiterverbreitet hat, muss offen bleiben. Nachrichten von solch dramatischen Lebenswenden wie der des Paulus haben sich wohl schon immer schnell herumgesprochen. (Schäfer 2004:79; zur Chronologie vgl. Riesner 1998:64-74)

Wahrscheinlich hatten die Galater auch von den Gegnern des Paulus - wenn auch verzerrt - Einzelheiten über dessen Vergangenheit gehört. Jedenfalls setzt die Verbreitung dieser Nachrichten übergemeindliche Verbindungen voraus.

17.Von den Gegnern wird gesagt, dass sie ein 'anderes Evangelium' propagieren (Ga 1:7). Aufgrund seiner Erstverkündigung kann Paulus das von ihm verkündete Evangelium im größeren Kreis der Gemeinden Galatiens als bekannte Größe voraussetzen. Ob dies neben seiner Erstverkündigung weitere übergemeindliche Verbindungen impliziert, ist unklar.

18.Zusammenfassung bei Bruce (1982:26); zum Zusammenhang vergleiche Schäfe (2004:13-75) ('Der Kontext der autobiographischen Aussagen des Paulus im Galaterbrief'); vergleiche auch Hengel und Schwemer (1997) und Riesner (1998), zur Chronologie vergleiche Campbell (2014).

19. Ähnlich Bruce (1982:90): '[I]t is plain from the sequel that others were circulating reports about him - reports which he regards as deliberately disparaging and which he rebuts from his own first-hand testimony'; vergleiche auch Seite 102.
In Galater 1:17 betont Paulus, dass er gerade nicht nach Jerusalem gezogen war, sondern seinen neuen Auftrag, Christus unter den Heiden zu verkündigen, sofort ausgeführt hat (vgl. Schäfer 2004:127-128). Hinter der Betonung seiner Unabhängigkeit steht wahrscheinlich die Zurückweisung von Gerüchten, die anscheinend weite Verbreitung gefunden hatten beziehungsweise deren Verbreitung und Kenntnisse bei den Galatern Paulus befürchtet: die führenden Leute in Jerusalem hätten ihn bald nach seiner Bekehrung in den Grundlagen des Evangeliums unterrichtet inklusive, so könnte berichtet worden sein, die weiterbestehende Forderung der Beschneidung. Aber Paulus hätte sich von dieser Vormundschaft und Autorisierung gelöst und mit seinem beschneidungsfreien Evangelium eine eigene Linie vertreten und damit eine Position, die jeder erkennbaren Autorisierung entbehrt. ${ }^{20}$ Die Verbreitung solcher Gerüchte beziehungsweise ihre Befürchtung setzt übergemeindliche Verbindungen voraus.

Nach einer Zeit in Arabien kehrt Paulus wieder nach Damaskus zurück. Je nach Einschätzung seines Wirkens in Arabien (kam es $\mathrm{zu}$ missionarischem Wirken und Gemeindegründungen durch Paulus? ${ }^{21}$ ) entstanden durch Paulus übergemeindliche Verbindungen zwischen den Gemeinden Arabiens, von Damaskus und Jerusalem. ${ }^{22}$

Wie ist der dezidierte Verzicht auf eine Reise nach Jerusalem zu werten? Wäre ein baldiger Besuch in Jerusalem eigentlich naheliegend gewesen, um Kontakt und Austausch mit den Aposteln zu suchen? Es ist unklar, ob solche Verbindungen nach Jerusalem zu diesem Zeitpunkt für alle Christen 'normal' waren und ob Jerusalem (zumindest in der Anfangszeit) eine besondere Stellung innehatte beziehungsweise ob dieser Hinweis lediglich der besonderen Biographie und Berufung des Paulus geschuldet ist. Wäre etwa zu erwarten, dass der ehemalige Verfolger der Christen Jerusalems dort Versöhnung sucht? ${ }^{23}$ Haben sich die Gegner des Paulus mit solchen Verbindungen nach Jerusalem gebrüstet? Falls dies der Fall ist oder impliziert ist, dass solche Kontakte die Regel sind, würde das übergemeindliche Verbindungen nach Jerusalem als die Norm bedeuten. Zumindest in der Frühzeit stünde nicht Paulus, sondern die dortigen Christen, im Mittelpunkt eines Netzwerks.

20.Nach Bruce (1982:95). Paulus hatte diese Darstellung bereits in Galater 1:12 verneint. Bruce skizziert die inhaltliche Widerlegung dieses Gerüchts auf Seite 95; vergleiche auch Seite 102.

21.Vergleiche die Diskussion bei Schnabel (2008:58-64) und Hengel und Schwemer (1997:106-126). Hengel und Schwemer gehen von missionarischem Wirken aus (S. 109), sprechen aber nicht von Gemeindegründungen (ähnlich Bruce 1982:97), bereits Ambrosiaster geht von Gemeindegründungen aus (Hengel \& Schwemer 1997:110); vergleiche auch Riesner 1998:256-262.

22.Schnabel (2008) vermutet, dass: 'The fact that Paul preached again in Damascus before he travelled to Jerusalem suggests that the Christian community of Damascus was the base of Paul's missionary activity in this region' (S. 64)

23.Von Lips (2016) schreibt: 'Jerusalem als Ausgangspunkt der urchristlichen Mission behielt weiterhin seine zentrale Bedeutung, die auch Paulus nie in Frage stellte. Darum war die hier erzielte Einigung mit den Aposteln und führenden Personen so wichtig' (S. 97). 
Erst drei Jahre später zieht Paulus nach Jerusalem, um Kephas kennenzulernen (Gal 1:18). ${ }^{24}$ Im Fokus steht die Aufnahme persönlicher Beziehungen mit den führenden Männern; von der Gemeinde ist nicht die Rede. ${ }^{25}$ Nur Jakobus wird erwähnt. Als Repräsentant des Flügels der Gemeinde, die sich besonders treu an das Gesetz hielt, wollte auch Jakobus diesen besonderen Gast kennenlernen und sich ein eigenes Urteil bilden (so Hengel \& Schwemer 1997:146). Jedoch agieren Petrus und Jakobus in diesen Begegnungen nicht als Privatpersonen, sondern als Repräsentanten der Jerusalemer Gemeinde. ${ }^{26}$ Paulus berichtet von einem längeren Aufenthalt sowie von der Gewährung und Annahme außerordentlicher Gastfreundschaft wohl durch Petrus (vgl. Hengel \& Schwemer 1997:144-150). Gastfreundschaft war eine wichtige Tugend im Urchristentum, 'and from the beginning was one of the basic presuppositions for the coherence of various Jesus communities and for the successful work of their itinerant missionaries' (Hengel \& Schwemer 1997:144, kursiv CS; vgl. Arterbury 2005). Nach Hengel und Schwemer ist seitens des Petrus mit persönlichem Interesse an diesem besonderen Besucher, der plötzlich in Jerusalem auftauchte, zu rechnen. Inhaltlich waren die fünfzehn Tage von regem Austausch bestimmt. Trotz der paulinischen Betonung der Unabhängigkeit seines eigenen Apostolats von den Aposteln (durch die Kontrastierung von seiner etwa dreijährigen Abwesenheit inklusive der Aktivitäten in Arabien und Damaskus und den lediglich 15 Tagen bei Petrus) ist es möglich, dass sich Paulus und Kephas während dieser 15 denkwürdigen Tagen nicht nur richtig kennenlernten, sondern auch von einander lernten:

The significance of each for the other was already known previously to both of them, since we may take it for granted that the former persecutor had already heard something about Peter when he was proceeding against the 'Hellenists' in Jerusalem and then again in Damascus. Otherwise he would not have looked him out at all. And that Peter, too, knew something about Paul follows not only from Gal 1:22 but also from the expulsion of the Hellenists, which certainly did not leave the 'Hebrews' and their leaders uninvolved. These two weeks were thus a good occasion for demolishing prejudices on both sides and building up a relative basis of trust. (Hengel \& Schwemer 1997:145-146)

Es ging also nicht nur um einen Höflichkeitsbesuch, sondern um eine wirkliche Begegnung, die in der weiteren Entwicklung des Urchristentums von Bedeutung war. $\mathrm{Zu}$ den Inhalten der Begegnung schreiben Hengel und Schwemer, dass dieser Prozess des Kennenlernens für Paulus weder darauf beschränkt war, von Kephas Informationen $\mathrm{zu}$ erhalten, noch auf Informationen über

24.Zu dieser Übersetzung vergleiche Schäfer (2004:128-129; zum Besuch S. 146-149) vergleiche auch Hengel und Schwemer (1997:133-150). Zur Bezeichnung Kephas vergleiche Williams (2015:30-45); zur Flucht aus Damaskus, Riesner (1998:75-89).

25.Dies betont Schäfer (2004): 'Paulus macht deutlich, nicht eine Gemeinde oder ein Gemeindegremium in Jerusalem besucht zu haben, sondern eine Einzelperson: Kephas. Ihn kennenzulernen, bezeichnet Paulus als Zweck seines mindestens primär privaten, möglicherweise sogar heimlich verlaufenen Jerusalembesuchs' (S. 146). Nach Hengel und Schwemer (1997:136) waren die Kontakte aus Gründen der Geheimhaltung angesichts möglicher Verfolgung beschränkt.

26. Hengel und Schwemer (1997:146) gehen davon aus, dass Petrus später der Gemeinde von diesem einzigartigen Besuch berichtet hat. das Wirken und die Lehre Jesu. Es ging um viel mehr. Zugleich gibt es keine persönliche Bekanntschaft, die nicht den Austausch von Informationen beinhaltet, im Besonderen, wenn sich die Begegnung über zwei Wochen hinzieht. Man darf annehmen, dass es Paulus weniger um persönliche Informationen über Petrus und seinen Charakter ging, sondern um dessen theologisches Denken, speziell um Christologie und Soteriologie, oder weniger abstrakt um den Inhalt seiner Verkündigung, die sicherlich auch die Erzählung der Worte und Taten Jesu beinhaltete. In anderen Worten: Jesus wird im Zentrum der Gespräche gestanden haben, etwa sechs Jahre nach dem Passafest, an dem er gestorben war - der irdische, gekreuzigte, auferstandene und erhöhte Jesus, der jetzt verkündigt wurde und der wiederkommen wird. Für beide, Kephas und Paulus, wurde Jesus und das Heil, das er gestiftet hatte, zur Mitte ihres Lebens. ${ }^{27}$ Dabei dürfte das Interesse nicht nur auf der Seite von Paulus gelegen haben, sonst hätte Kephas ihn wohl kaum für 15 Tage bei sich aufgenommen. In nicht geringerem Maß dürfte es auch Kephas ein Bedürfnis gewesen sein, Paulus und seine Botschaft gründlich kennenzulernen:

In other words, Paul certainly reported his call by the Risen Christ and his missionary preaching $[\ldots]$ and at the same time spoke - as a former Pharisaic scribe - of his understanding of the prophetic promise and the Torah and - inseparably connected with this - of his gospel, which he had received personally from the Risen Christ. If all this had not been discussed, they could have parted earlier. [...] The duration of the hospitality indicates the intensity of the exchange. In both cases there will have been a charismatic curiosity brought about by the Spirit. (Hengel \& Schwemer 1997:146-147) ${ }^{28}$

Hengel und Schwemer fragen $\mathrm{zu}$ Recht nicht nur, welche Traditionen Paulus in diesen Tagen von Kephas empfangen haben könnte, sondern diskutieren auch mögliche Auswirkungen dieses Besuchs auf Petrus. ${ }^{29}$

Durch diesen intensiven Besuch ist Paulus fest mit den Jerusalemern - vielleicht auch über die 'Säulen' hinaus verbunden. Hinter dem durch eine Schwurformel (Gal 1:20 'Was ich euch aber schreibe - siehe, Gott weiß, ich lüge nicht')

27.Zu den Inhalten des Austausches mit Petrus schreibt Schäfer (2004), dass Kephas wegen seiner engen Beziehung zu Jesus für Paulus interessant sein dürfte. Paulus dürfte die fünfzehn Tage dazu genutzt haben: 'alles das über den an ihm selbst offenbar gewordenen Sohn (vgl. Gal 1:16) von Kephas zu erfahren, was eben nicht offenbar gewordenen Sohn (vgl. Gal 1:16) von Kephas zu erfahren, was eben nicht
Gegenstand der Offenbarung oder des bis dahin erworbenen Wissens, aber über Gegenstand der Offenbarung oder des bis dahin erworbenen Wissens, aber über
jenen gleichwohl für ihn zugänglich war. Wie hätte das frühere distanzierte jenen gleichwohl für ihn zugänglich war. Wie hätte das frühere distanzierte
Interesse des Verfolgers oder gar das Offenbarungserlebnis zu einem für Paulus befriedigenden Wissensstand bezüglich Leben, Wirken, Sterben und ersten befriedigenden Wissensstand bezüglich Leben
Erscheinungen Jesu führen sollen?' (S. 148).

28.Sie führen den Bericht aus 1Korinther 15:2-8 (eine Mischung aus Bekenntnis, Katechese und Geschichte) zumindest in Teilen auf diese Begegnung zurück. Dort betont Paulus seine Übereinstimmung mit den Aposteln: 'Es seien nun ich oder jene: so predigen wir, und so habt ihr geglaubt' (1Kor 15:11). Diese Beobachtung banishes the view so popular today that there were many contradictory "kerygmata" and disputatious groups at the beginning of earliest Christianity to the realm of modern mythologizing' (S. 147).

29. Hengel und Schwemer (1997). Auf Seite 149 schreiben sie: 'In any case, these intensive fourteen days in Jerusalem, the brevity of which should not constantly be emphasised [...] were not unimportant for the further fortunes of the young movement' (pp. 147-149). Nach Apostelgeschichte 9:26-30 kam der Aufenthalt

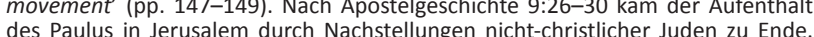
Saulus wird von den Brüdern beziehungsweise Petrus in Sicherheit gebracht: 'His Saulus wird von den Brüdern beziehungsweise Petrus in Sicherheit gebracht: 'His
host will certainly have continued to feel responsible for the safety of his endangered (and dangerous) guest' (S. 150) 
beteuerten Hinweis auf Dauer und Inhalte des Besuchs in Jerusalem liegt die dezidierte Zurückweisung des Vorwurfs:

Paulus hatte keinen in irgendeiner Form 'offiziellen' Kontakt zu dem Gremium der Apostelinnen und Apostel in Jerusalem zur Bestätigung oder Auslegung seines in einem Offenbarungsgeschehen grundgelegten Auftrags (vgl. Gal 1:16c, 17a, 19a). (Schäfer 2004:147) $)^{30}$

Das Wissen um den relativ späten ersten Besuch des Paulus in Jerusalem nach seiner Berufung kann daher nicht gegen die Position des Paulus angeführt werden. ${ }^{31}$ Solche Kontakte nach Jerusalem zur Bestätigung und zum inhaltlichen Abgleich der Verkündigung werden in dieser Argumentation als Notwendigkeit und Norm vorausgesetzt. Damit sind wichtige Motive für persönliche und übergemeindliche Verbindungen benannt.

Nach seinem Aufenthalt in Jerusalem reist Paulus nach Syrien und Kilikien, um dort missionarisch zu wirken und Gemeinden zu gründen (Gal 1:21). ${ }^{32}$ Aufgrund der folgenden Angabe (siehe unten) fasst Bruce (1982:103) das dortige Wirken des Paulus und seine übergemeindliche Wirkung knapp zusammen: '[A]t any rate, enough was happening for news of Paul's activity to get back to Judaea.' Durch ihn - und eventuell andere Christen, die Paulus begleitet haben oder aus diesen Gebieten stammten entstehen Verbindungen dieser Gemeinden $\mathrm{zu}$ den Christen in Damaskus, Arabien und Jerusalem und dieser Gemeinden untereinander.

Trotz des Aufenthalts in Jerusalem war Paulus den Gemeinden in Judäa von Angesicht unbekannt (Gal 1:22; vgl. vgl. Röm 15:19: 'von Jerusalem bis nach Illyrien'?). ${ }^{33}$ Allerdings hatten diese Gemeinden von der Bekehrung und dem folgenden Dienst des Paulus gehört: 'Der uns früher verfolgte, der predigt jetzt den Glauben, den er früher zu zerstören suchte' (Gal 1:23; zum Gerücht vgl. Schäfer 2004:155-157). Die periphrastische Konstruktion $\mu$ óvov $\delta \grave{\varepsilon}$

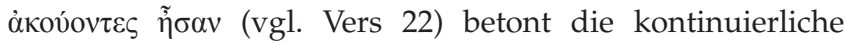
beziehungsweise iterative Weise der genannten Aktion: immer wieder wiederholt hatten sie von seiner Lebenswende und seinem Dienst gehört. ${ }^{34}$ Aufgrund dieser Nachricht

30.Weiter betont Schäfer, dass die Angaben des Paulus bezüglich Zeitpunkt und Dauer seines Jerusalemaufenthalts genau sind. Damit waren sie für die Adressaten zumindest prinzipiell überprüfbar (S. 147).

31.Nach Hengel und Schwemer (1997) weist Paulus damit noch andere Vorwürfe zurück: 'It had probably been spread around by the Judaisers in Galatia that he initially had close connections with the community in Jerusalem and its leading body and was dependent on them' (S. 133). Wenn dies zutrifft, zeigt die Widerlegung des Paulus, dass solche Verbindungen als normal angesehen wurden, Widerlegung des Paulus, dass solche
zumindest jedoch plausibel waren.

32.Vergleiche Hengel und Schwemer (1997:156-158, 260-267) und Riesne (1998:264-268). Riesner geht aufgrund von Apostelgeschichte 15:41 von der Gründung von Gemeinden aus. Nach Riesner könnte Paulus während der Zeit in Syrien und Kilikien auch bis nach Kappadozien vorgestoßen sein (S. 267-268).

33.Zur rhetorischen Funktion dieser Aussagen vergleiche Moo (2013:112); zur Bedeutung von Judäa Seite 113. Nach Schäfer (2004:152) wird 'Judäa' dabei 'von Paulus wahrscheinlich als Bezeichnung für ganz Palästina unter Einschluss von Galiläa, Samaria und Peräa verwendet.'

34.Vergleiche Von Siebenthal (2011:338). Vergleiche auch die Imperfektform in Vers 24 (zur iterativen Bezeichnung der Vergangenheit durch Imperfektformen vgl. Von Siebenthal 2011:324). Bruce (1982) übersetzt daher: '[T]hey glorified God on my account - every time they heard such news (imperfect tense).' Bruce schreib ferner: 'As some members of those churches received the news, they would pass it preisen sie Gott (vgl. Schäfer 2004:152-159). Sowohl das Wissen des Paulus um diese Gemeinden und um das, was sie gehört hatten, als auch die Verbreitung der Nachricht von seiner Lebenswende unter den Gemeinden setzen übergemeindliche Verbindungen in Judäa oder über Judäa hinaus bis nach Damaskus, Syrien und Kilikien voraus beziehungsweise von dort zurück nach Judäa. Sie sind die Grundlage für das Lob Gottes.

Nach Schäfer (2004:153) ist die einfachste Erklärung der persönlichen Unkenntnis des Paulus die Annahme, dass er eine Reiseroute vermied, die ihn $\mathrm{zu}$ schon bestehenden christlichen Gemeinden geführt hätte. Neben einer Schiffsreise von Cäsarea aus ist an den Weg durch den Jordangraben über Skythopolis oder als östlichste Route der alte Königsweg über Philadelphia und Gerasa zu denken. Allerdings kann die andere Interpretation, nach der Paulus Städte mit christlichen Gemeinden durchzogen habe, ohne sich dort aufzuhalten, nicht ausgeschlossen werden. ${ }^{35}$

$\mathrm{Zu}$ fragen ist, ob Paulus eine solche Route wählen musste, um etwaigen Nachstellungen aus dem Weg zu gehen oder um den Gemeinden, bei denen er noch nicht voraussetzen konnte, dass sie von seiner Lebenswende gehört hatten, den Schrecken seines Erscheinens zu ersparen. Womöglich wollte Paulus durch seinen Verzicht auf Besuche auch Schwierigkeiten für die Gemeinden vermeiden. Doch setzt die Erklärung der mangelnden persönlichen Kenntnis voraus, dass entsprechende Kontakte wohl die Regel und nicht die Ausnahme waren.

Nach Schäfer (2004) ist das Gerücht von der Lebenswende des Paulus im Raum der Gemeinden Syriens und Kilikiens zu verorten, da nur Christen in diesem geografischen Raum Paulus in seiner neuen Rolle als Verkündiger des Evangeliums erlebt haben:

In diesem Raum müssen [...] Jesusjünger in ihm ihren ehemaligen Verfolger erkannt haben. Eine derartige Nachricht, wie sie von Paulus angeführt wird, hat demnach ihren ersten Erfahrungshintergrund bei [...] Jesusjüngern, die sich in Syrien oder Zilizien aufhielten. (S. 155)

Schäfer beobachtet ferner, dass Paulus von der Verbreitung der genannten Nachricht bis nach Judäa ausgeht. Auch dort und nicht nur in den weiter nördlichen Gebieten wird seine Lebenswende unter den christlichen Gemeinden bekannt. Die Aussage in Galater 1:23 weist also auf Verbindungen zwischen den Gemeinden des syrisch-kilikischen und des

on to others: Have you heard? Our former persecutor [...] is preaching the gospel which he once tried to destroy' (S. 105).

35.Vergleiche auch die Diskussion auf Seite 154. Nach Schäfer ist unter den Erklärungen dieses Sachverhalts die Annahme am plausibelsten, dass der Glaubensgeschwistern war und zudem vielleicht frühere Freunde fürchten musste: 'Elaubensgeschwistern war und zudem vielleicht frühere Freunde furchten musste: 'Ein anderes Erklarungsmodell geht davon aus, dass Paulus ja schon früh seine konkrete Sendung zu den [...] Heiden (vgl. Gal 1:16) umsetzen wollte und umgesetz hat (vgl. Gal 1:17), die Verkündigung in den palästinischen Gemeinden abe ordringlich auf [...] Juden ausgerichtet war. Vielleicht hat Paulus sich von solc einem Kontakt keine Anregungen für die Form von missionarischer Wirksamkeit erhofft, die ihm selbst vorschwebte' (S. 154). Schäfer (2004) beleuchtet dort auch die rhetorische Funktion dieses Hinweises. 
judäischen Raumes hin. Als vermutliche Überbringer der genannten Nachricht:

$[K]$ önnen entweder Mitglieder einer Gemeinde in Syrien oder Zilizien gelten, die sich aus irgendeinem Grunde auf den Weg nach Palästina begeben haben, oder es handelt sich umgekehrt um Angehörige einer palästinischen Gemeinde, die den entsprechenden Erfahrungshintergrund aufweisen und von einem Aufenthalt in den weiter nördlich gelegenen Gebieten zurückkehren. (S. 155-156)

Allerdings liegt es näher, das Personalpronomen am Anfang von Vers 23 ('Sie hatten nur gehört [...]') auf die Gemeinden in Judäa zu beziehen.

\section{Galater 2}

In Galater 2:1-2 schreibt Paulus, dass er aufgrund einer Offenbarung nach Jerusalem kam. ${ }^{37}$ Der Inhalt der Offenbarung wird nicht genannt. Paulus wurde also von niemandem nach Jerusalem zitiert, sondern kam aufgrund der Offenbarung aus freien Stücken (vgl. Moo 2013:123). Vorher war von Syrien und Kilikien die Rede (Gal 1:21), so dass anzunehmen ist, dass er von dort kam. Daher schließen Hengel und Schwemer, dass Paulus und Barnabas nicht nur als Repräsentanten der Gemeinde in Antiochien in Jerusalem erschienen, sondern als Repräsentanten aller Gemeinden in Syrien und Kilikien, das heißt, wahrscheinlich all der Gemeinden, in denen sie gewirkt hatten und die ihre Position in der Frage der Beschneidung und des rituellen Gesetzes teilten. ${ }^{38}$ Paulus kam mit Barnabas und Titus, das heißt auch, seine Mitarbeiter sind an den so entstehenden Verbindungen beteiligt. $^{39}$

Im Rahmen dieses Besuchs fand ein Austausch über den Inhalt des Evangeliums statt; '[U]nd besprach mich mit ihnen über das Evangelium' (Gal 2:2). ${ }^{40}$ Paulus berichtet dies in einem Kontext, in dem er seine Unabhängigkeit von Jerusalem betont. Hier dienen Verbindungen zwischen Repräsentanten der Gemeinden in Damaskus, Arabien,

36.Nach Schäfer (2004:156-157) ist es historisch plausibel, dass die Nachricht von seiner Lebenswende und seinem christlichen Engagement schon früh bis zu vielen judäischen Gemeinden und darüber hinaus verbreitet wurde.

37. Moo betont, dass die knappen Informationen über den Besuch und die Argumentation voraussetzen, dass die Galater mit den Ereignissen bei diesem Jerusalembesuch vertraut waren. Sie hatten davon entweder von Paulus selbst erfahren oder von den Gegnern die Paulus, die auf dieses Ereignis verwiesen ('Probably because the meeting has already been brought up by the agitators for their own purposes, Paul assumes that the Galatians know quite a lot about it" (2013:124) und es aus der Sicht des Paulus falsch darstellten.

38. Hengel und Schwemer (1997:261). Aus der Formulierung 'mit Barnabas' und 'unter Mitnahme von Timotheus' und bei Beschränkung auf die Angaben des Galaterbriefs 'motiviert allein diese Ausdrucksweise in Gal 2:1 die gängige Galaterbriefs 'motiviert allein diese Ausdrucksweise in Gal 2:1 die gängige Meinung, in ihm und in Barnabas [...] Delegierte einer Gemeinde zu sehen', so
Schäfer (2004:168). Sie geht davon aus, dass die drei Männer aus Antiochien nach Schäfer (2004:168). Sie geht davon
Jerusalem kamen (S. 169-170).

39.Zu Paulus und Barnabas vergleiche Hengel und Schwemer (1997:205-221), Kollmann (1998) und Öhler (2002). Hengel und Schwemer sehen in Barnabas einen Garanten dieser Verbindung zwischen Jerusalem und Antiochien. Es se bemerkenswert, dass gerade dieser Mann, der aufgrund seiner Herkunft eine besondere Verbindung mit der Gemeinde in Jerusalem hatte, nun für längere Zeit der Missionspartner des Paulus wurde. Nach dem Bruch zwischen ihm und Paulus der Missionspartner des Paulus wurde. Nach dem Bruch zwischen inm und Paulus wurde Barnabas durch Silas-Silvanus aus Jerusalem ersetzt, auch wenn dies nur fü eine Reise von ungefähr zweieinhalb Jahren der Fall war (1997:204-205). Zu Bedeutung des Barnabas in den Gesprächen in Jerusalem vergleiche Seite 209; zu
Titus vergleiche Dunn (2009:478-479) und Von Lips (2016:91-122).

40.Zur Aussage 'damit ich nicht etwa vergeblich liefe oder gelaufen wäre' vergleiche Hengel und Schwemer (1997:208).
Syrien und Kilikien und Jerusalem ${ }^{41}$ dem Austausch über Inhalte der Botschaft und der innerkirchlichen Anerkennung und Abstimmung der Arbeitsbereiche.

Die Reise - aufgrund einer Offenbarung - und die Anerkennung seines Dienstes in Jerusalem sowie die dortige Entscheidung zeigen, dass Paulus zumindest zu diesem Zeitpunkt der Gemeinde in Jerusalem eine besondere Stellung und Autorität zumisst. Paulus erkennt die legitime Autorität und Bedeutung der Jerusalemer Apostel an und ist überzeugt, dass ihr Einverständnis mit seinem Verständnis des gesetzesfreien Evangeliums wichtig ist (vgl. Moo 2013:119).

In Jerusalem wurden Paulus und seinen Begleitern keine weiteren Bedingungen auferlegt. Selbst der Heidenchrist Titus wurde nicht gezwungen, sich beschneiden zu lassen (Gal 2:3). Paulus musste die Gemeinschaft mit ihm in Jerusalem nicht unterbrechen oder gar ganz aufkündigen. ${ }^{42}$ Titus wurde in dieser Situation zum Modellfall. Auch wenn seine Gegenwart in Jerusalem das zu verhandelnde Problem nicht provoziert hat (vgl. Gal 2:11-14), mag sie zu dessen Konkretisierung beigetragen haben: es geht nicht nur um die grundsätzliche Bedeutung des Gesetzes (müssen Heidenchristen beschnitten, d.h. Juden werden?), sondern auch um eine Grundsatzentscheidung bezüglich der Träger der Mission:

Und dass kein Zwang auf Titus ausgeübt wurde besagt dass die Führenden der Jerusalemer Gemeinde die Position des Paulus anerkannten, eine Grundsatzentscheidung, die es Paulus ermöglicht, eben einen unbeschnittenen getauften Heiden als Mitarbeiter zu nehmen. (Von Lips 2016:100)

Wie später in Antiochien haben auch in Jerusalem übergemeindliche Verbindungen zur Klärung und Entwicklung urchristlicher Theologie beigetragen.

Bei den in Galater 2:4 erwähnten 'falschen Brüdern', die sich in die erwähnten Beratungen 'eingeschlichen' haben, ist nicht klar, ob sie aus Jerusalem stammen oder von anderswoher nach Jerusalem gekommen waren. Für letztere Option könnte die Angabe in Vers 5 sprechen: die Ereignisse in Jerusalem haben Implikationen für die Christen Galatiens: '[D]amit die Wahrheit des Evangeliums bei euch

41.Nach Schäfer (2004) handelt es sich bei den Gesprächspartnern des Paulus um Vertreter der Jerusalemer Gemeinde. Nach dem Kontext Gal 1:17-19 und der bisher erschlossenen Zielrichtung des Rückblicks sei es naheliegend, bei den aủtoĩ von Galater 2:2 an die Jerusalemer Apostel von Galater 1:17(a) 19 zu

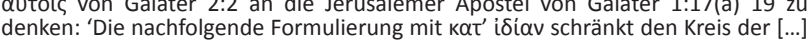

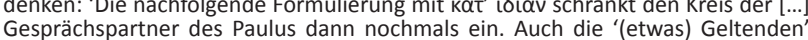
Gesprächspartner des Paulus dann nochmals ein. Auch die '(etwas) Geltenden'
scheinen zwar zu den [...] Aposteln zu gehören, stellen aber nur eine Teilgruppe dar und ragen unter jenen noch einmal in besonderer Weise hervor' (S. 170-171). Die

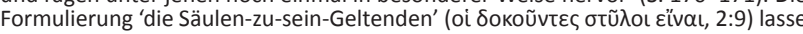
konkret an Jakobus, Kephas und Johannes als Gesprächspartner des Paulus (und des Barnabas) denken. trifft dies zu, würde auch Johannes zur Gruppe der Aposte in Jerusalem gezählt werden: 'Unter 'Jakobus' sollte hier wie in Galater 1:19 (und Gal 2:12) der Herrenbruder verstanden werden. Das Treffen in Jerusalem wäre den paulinischen Sprachgebrauch zugrunde gelegt, ein ,Apostelkonvent' im eigentlichen Sinne des Wortes' (Schäfer 2004:171).

42.Mit Blick auf die in Galater 2:12 erwähnte Gruppe vermutet Schäfer (2004:232), dass sie für sich eine ähnliche Sonderrolle beansprucht habe, wie sie Paulus seinerzeit in Jerusalem beansprucht hatte. Dort konnten der Heide Titus und er weiterhin ihre enge Gemeinschaft pflegen. Ihre 'Freiheit' (Gal 2:4) wurde von den weiterhin ihre enge Gemeinschaft pflegen. Thre 'Freiheit' (Gal 2:4) wurde von den Antiochien ihre eigene, observante Praxis fortfuhren dürfen?' 
bestehen bliebe. ${ }^{43}$ Kommen sie von außen, sind sie ein weiterer Hinweis auf übergemeindliche Verbindungen. Auch wenn ihr Vorgehen negativ konnotiert ist ('um unsere Freiheit auszukundschaften, die wir in Christus haben, und um uns zu knechten'), zeigt ihr Vorgehen die Absicht, gemeindeübergreifend anderen Christen gegenüber (in diesem Fall Paulus und seine Begleiter und durch sie über ihre Gemeinden) eine Kontrollfunktion auszuüben. ${ }^{44}$

Gegenüber diesen Erkundigungen und Forderungen steht die Anerkennung der führenden Jerusalemer (V. 6-9; 'mir haben die, die das Ansehen hatten, nichts weiter auferlegt', V. 6). Das Evangelium des Paulus war also nicht ergänzungsbedürftig, wie seine Gegner in Galatien argumentiert haben dürften. Ferner kommt es zur Absprache der Wirkungsbereiche (V. 9). Anerkennung der Verkündigung und Absprachen von Arbeitsbereichen sind aufgrund übergemeindlicher Verbindungen möglich und zeigen die inhaltlich-theologische und praktische Bedeutung solcher Verbindungen. Die Galater hatten von den Ereignissen in Jerusalem und den dortigen Beschlüssen gehört, beziehungsweise sie erhalten jetzt die Richtigstellung und Perspektive des Paulus auf die Ereignisse. All das wird den Christen Galatiens von Paulus mitgeteilt. Durchweg im autobiografischen Teil des Briefs vertiefen Informationen übergemeindliche Verbindungen, diese wiederum führen zum Austausch von Informationen. Dass diese Informationen immense Implikationen vor Ort haben (können), liegt auf der Hand. Dieser Informationsaustausch, sowohl durch die Gegner als auch durch Paulus, ist ein wesentlicher Bestandteil übergemeindlicher Verbindungen.

Die einzige erhobene Forderung, nämlich der Armen zu gedenken (Gal 2:10), bleibt vage. Wenn es sich allgemein um Arme im abgesprochenen Wirkungsbereich des Paulus handelt, trägt der Hinweis zu unserer Fragestellung nichts bei. Wenn es sich allerdings spezifisch um die Armen der Jerusalemer Gemeinde handelt (ohne dass dies explizit erwähnt wird), dürfte in dieser Forderung der Ursprung der späteren Kollekte des Paulus liegen. Unabhängig von der Datierung des Galaterbriefs ${ }^{45}$ ergibt sich eine weitere Perspektive. Ist der Besuch in Jerusalem mit dem sogenannten Hungerhilfebesuch der Apostelgeschichte (11:27-30) gleichzusetzen (und nicht eine paulinische Reflektion auf das sogenannte Apostelkonzil in Apg 15), wäre die Aufforderung an die Armen zu denken, eine Aufforderung, die gerade geschehene Hilfeleistung durch die Gemeinde in Antiochien fortzuführen, im Sinne von: '[N]ur dass wir weiterhin der Armen gedächten.' Damit ständen hinter dem

43.Vergleiche dazu Nanos (2005). Schäfer (2004:193) erwägt ein Ausspionieren von Gemeindeverhältnissen an einem anderen Ort und vermutet eine Bezugnahme auf antiochenische Verhältnisse.

44.In der Apostelgeschichte erscheint diese Kontrollfunktion an mehreren Stellen positiv, aber auch negativ. Dort ist sie deutlich mit übergemeindlichen Verbindungen verbunden.

45.Zur kontroversen Diskussion vergleiche Moo (2013:2-18). Auch be einer Spätdatierung des Briefs (d.h., Empfänger in Nordgalatien bzw. Landschaftshypothese) kann sich Galater 2:10 immer noch auf die in Apostelgeschichte 11 erwähnte Sammlung beziehen, auch wenn sie in der Vergangenheit stattgefunden hat. 'wir' nicht nur Paulus, Barnabas und Titus, sondern die Gemeinde in Antiochien, als deren Repräsentant Paulus und seine Begleiter nach Jerusalem gekommen waren (Apg 11:30: 'und schickten sie zu den Ältesten durch Barnabas und Saulus') und - zumindest in einer gewissen Phase - sich verstanden haben dürften (so zumindest die Darstellung der Apostelgeschichte). Dass Paulus auch die Gemeinden Galatiens an der Kollekte beteiligen wollte, zeigt 1 Korinther 16.1: 'Wie ich in den Gemeinden in Galatien angeordnet habe, so sollt auch ihr tun. ${ }^{46}$ Die Beteiligung zweier Christen aus Südgalatien an der Kollektendelegation (Apg 20:4: 'und Sekundus und Gajus aus Derbe') dürfte ein Hinweis sein, dass zumindest einige Galater der Aufforderung des Apostels gefolgt waren. ${ }^{47}$

Der spannungsvolle Abschnitt Galater 2:1-10 bewegt sich zwischen den Polen der Betonung der Unabhängigkeit des Paulus und der Anerkennung durch andere Christen in Jerusalem. ${ }^{48}$ Diese Dialektik muss stets mitbedacht werden. Bei aller Betonung seiner Unabhängigkeit, weiß sich Paulus anderen Christen verbunden. In der von Paulus angestrebten und empfangenen Anerkennung zeigt sich etwas von der Bedeutung übergemeindlicher Beziehungen beziehungsweise der Zugehörigkeit zu einem größeren Ganzen.

Auch für das folgende Ereignis, den sogenannten 'antiochenischen Zwischenfall', liegt nur die Schilderung und Interpretation des Paulus vor. ${ }^{49}$ Der zuvor in Jerusalem verortete Kephas (2:7-9) kommt als weiterer Schritt seines Wirkens über Jerusalem hinaus (vgl. Apg 9:32-12:17) nach Antiochien (Gal 2:11; vgl. Apg 8:14-25)..50 Dorthin muss Paulus nach der Begegnung in Jerusalem (zurück)gereist sein. Nach seiner Einbindung in die Gemeinden von Damaskus und Jerusalem, präsentiert Paulus sich als Teil der antiochenischen Gemeinde. Es bleibt unklar, ob er dort aus eigener Initiative oder im Auftrag der Gemeinde handelte. Seine Verankerung in der dortigen Gemeinde nach dem Bild der Apostelgeschichte macht letztere Möglichkeit zumindest möglich, wenn nicht plausibel. Paulus verweist hier auf gegenseitige Besuche von ihm und anderen in Jerusalem und von Petrus in Antiochien, auch wenn sie anders motiviert gewesen sein mochten (geht es um Zuständigkeitsfragen, Kontrolle, Anbindung an Jerusalem?; die Motive des Petrus werden nicht erwähnt). Durch Repräsentanten entstehen übergemeindliche Verbindungen.

46.Schäfer (2004:222) geht von einer Spätdatierung des Galalaterbriefs aus und schließt aus 1 Korinther 16:1, dass die Galater um das in Galater 2:10 beteuerte schließt aus 1 Korinther 16:1, dass
Engagement des Paulus wussten.

47.Mit dem Hinweis auf den Ursprung der Kollekte in einer Forderung der Jerusalemer zapft Paulus deren Autorität für sein groß angelegtes Spendenprojekt an. Es geht Paulus nicht darum, sich zu bereichern oder seinen Ruf aufzupolieren, sondern er kommt lediglich treu einer Aufforderung nach. Aufgrund der Aufforderung der Jerusalemer darf mit der Annahme der nicht unumstrittenen Kollekte in Jerusalem gerechnet werden.

48.Schäfer (2004:176) betont die klaren Grenzen dieser Anerkennung.

49.Vergleiche Driedger (2015) und Dunn (2009:470-482). Dunn skizziert auch knapp die im Hintergrund der Ereignisse stehende, angespannte politische Situation in Judäa (S. 480-481).

50.Zur Entstehung der Gemeinde in Antiochien vergleiche Hengel und Schwemer (1997:178-205) und Riesner (1998:108-114). Nach Schäfer (2004:226) handelt es sich vermutlich um den ersten Aufenthalt des Kephas in einer Gemeinde des syrisch-kilikischen Raums. Zumindest wird nichts anderes vermerkt. Auch der Anlass der Reise des Kephas nach Antiochien wird nicht genannt. 
Bezüglich der Verbindungen zwischen Jerusalem und Antiochien unterstreichen Hengel und Schwemer, dass die Entwicklung in der ersten mehrheitlich heidenchristlichen Gemeinde in Antiochien niemals ganz von den Ereignissen in Jerusalem getrennt werden kann. Als früheste literarische Hinweise zeigen insbesondere die echten Paulusbriefe, dass keine wirklichen Separationsbestrebungen in der frühen Phase der neuen messianischen Gruppierung aufgezeigt werden können. Es mag Spannungen und unterschiedliche Parteiungen gegeben haben. In einer enthusiastischen Bewegung wie dem frühesten Christentum können diese nie ganz vermieden werden. Aber es gibt keine Hinweise auf definitive Trennungen und Spaltungen. Sie hätten die neue Botschaft von Anfang an unglaubwürdig gemacht (so Hengel \& Schwemer 1997:204):

The one Lord, the one Spirit and the Community as the 'one body of Christ', made cohesion, not least with the 'mother community' in Jerusalem, an obligation. If this is true for the Paul of the letters during his mission in Macedonia, Achaea and the province of Asia despite all the disappointment and deeper theological differences, it is even more true for Antioch and the other communities in Syria. [...] Paul took this bond, which linked him to Jerusalem and was probably obligatory for all Christian communities [...] very seriously [...] although the more time went on, the more trouble it caused him. (pp. 204-205)

Während eines wohl längeren Aufenthalts in Antiochien hielt Petrus zunächst Tischgemeinschaft mit Heidenchristen (Gal 2:12), passte sich also der Praxis vor Ort an (vgl. Schäfer 2004:229). Übergemeindliche Verbindungen fordern schon im Urchristentum Flexibilität und führen zu neuen Erfahrungen (vgl. Apg 10:1-11,18).

Als aber 'einige von Jakobus' nach Antiochien kamen, ${ }^{51}$ zog Petrus sich zurück und sonderte sich von der Tischgemeinschaft mit den Heidenchristen $a b,{ }^{52}$ 'weil er die aus dem Judentum' fürchtete.

Das Verhalten des Petrus, der als Gast nach Antiochien gekommen war, hatte auch Folgen für die anderen Judenchristen dort: 'Und mit ihm heuchelten auch die anderen Juden, so dass selbst Barnabas' verführt wurde, mit ihnen zu heucheln.' Barnabas Verhalten wird von Paulus scharf kritisiert. Diese Kritik hat in der Forschung einen Schatten auf Barnabas geworfen. Man kann das Verhalten von Petrus, Barnabas und anderen auch als Ausdruck seiner nach wie vor starken Verbindungen zu den Christen Jerusalems werten. Nach Hengel und Schwemer (1997:215) wird die relative starke Verbindung des Barnabas nach Jerusalem auch in Galater 2:13 sichtbar, als Barnabas, wie die anderen Judenchristen, durch die Furcht des Kephas vor der Abordnung des Jakobus dazu 'verführt wurde', die

51.Dunn (2009:479) schreibt zur Bezeichnung 'von Jakobus': "[w] wich can hardly indicate other than that the authority of James was behind the newcomers, either explicitly or implicitly, either formally or in effect claimed by them.'

52.Zum wahrscheinlich pharisäischen Hintergrund des hier verwendeten Absonderungsbegriffs vergleiche Dunn (2009:472); zum Ausdruck 'heidnisch leben' in Galater 2:14 vergleiche Seiten 472-473. Dunn beleuchtet auch die instruktiven Parallelen in 4QMMT (S. 476-478) eucharistische Tischgemeinschaft mit den Heidenchristen abzubrechen:

Paul's form of expression suggests that this 'defection' took place after some hesitation, but evidently the tie to Jerusalem and the solidarity with the other Jewish Christians in Antioch were stronger than the mission shared with Paul over long years which also had a theological basis. (S. 215)

Der Ausdruck 'die aus dem Judentum' bezeichnet die Leute des Jakobus als Judenchristen. Mit der Formulierung 'einige von Jakobus' bleibt unklar, ob sie wirklich von Jakobus gesandt wurden (wenn ja, mit einem spezifischen Auftrag? Visitation, Klärung, Ankündigung der problematischen Folgen dieser Tischgemeinschaft von Juden und Heiden in Antiochien für die Gemeinde in Jerusalem) oder ob sich die Besucher zu Unrecht auf Jakobus berufen hatten, etwa um ihre eigene Autorität zu stärken. ${ }^{53}$

Zur Situation, die die Gruppe aus Jerusalem vorfand und der gegenüber sie sich positionieren musste, und den verschiedenen Optionen, die sie dabei hatte, beobachtet Schäfer, dass die Neuankömmlinge die gleiche Reisebewegung vollzogen wie zuvor Kephas (von Jerusalem nach Antiochien) und in Antiochien wohl auch vor das gleiche Problem wie er gestellt wurden. Neben diesen Gemeinsamkeiten gibt es jedoch signifikante Unterschiede: Während sich Kephas in die Praxis vor Ort eingefügte, konnten sich diese Judenchristen:

offenbar nicht dazu durchringen, Tischgemeinschaft mit den [...] Heidenchristen aufzunehmen. Als Gruppe-im Numeruswechsel liegt der einzige Unterschied zwischen Galater 2:11a und 2:12b - steht ihnen eben auch die Möglichkeit offen, beim Mahlhalten unter sich zu bleiben. Vermutlich nehmen die betreffenden $[\ldots]$ Jünger einfach für sich in Anspruch, weiterhin gesetzesobservant leben zu wollen und zu sollen. (Schäfer 2004:231-232) (44 $^{54}$

Die Flexibilität und Bereitschaft des Petrus waren also keineswegs selbstverständlich. $\mathrm{Ob}$ die Jerusalemer Besucher entsprechende Forderungen an Petrus und andere jüdische Christen gestellt haben, wird nicht erwähnt. ${ }^{55}$

53.Nach Schäfer (2004:230) handelt es sich um eine Gruppe von jüdischen JesusJüngern aus dem Umfeld des Jakobus, 'der Führungspersönlichkeit in der Jerusalemer Gemeinde zur vorausgesetzten Zeit.' Gegenüber einem Rückschluss auf eine quasi 'offizielle' Beauftragung dieser Leute durch Jakobus, 'etwa gar noch zur "Visitation" der Verhältnisse in Antiochien' rät Schäfer zu Recht zur Vorsicht: 'Das vorgetragene Verständnis des Jerusalemer Abkommens als einer Übereinkunft nach dem Prinzip der Arbeitsteilung mit verschiedenen Zuständigkeiten bzw. kch dem Prinzip der Arbeitsteilung mit verschiedenen Zuständigkeiten bzw. Verantwortungsbereichen spricht eher gegen eine solche Interpretation. Wohl legt die ungeschützte paulinische Formulierung die Annahme einer Verwandtschaft de Auffassungen derer "von Jakobus" mit denen von Jakobus nahe' (S. 231) vergleiche
Deines (2017:162-165).

54.Schäfer (2004). Zur Motivation der Gruppe vergleiche Seite 232.

55.Für Paulus sind sie wie Kephas in Antiochien nur Gäste, die sich der Praxis vor Ort anzupassen haben; vergleiche Schäfer (2004:230, 234-243). Anscheinend lässt Paulus die ihm und Titus in Jerusalem zugestandene Freiheit gegenüber den Gästen aus Jerusalem in Antiochien gelten, interveniert aber, als er die Praxis der dortigen Gemeinde gefährdet sieht und - weit über die konkrete Praxis hinaus sein Verständnis des Evangeliums. Die Reaktion der Heidenchristen und mögliche ihnen gegenüber erhobene Forderungen, sind nicht im Blick. Freilich stand die Rücknahme der gesetzesfreien Heidenmission in Antiochien zu keinem Zeitpunkt zur Debatte. Hatte Paulus überreagiert? Vergleiche auch Dunn (2009:474-475) dur Debatte. Hatte Paulus überreagiert? Vergleiche auch Dunn (2009:474-475), dort auch eine sympathische Interpretation des Verhaltens des Petrus und seine Beweggründe (S. 481-482): 'All in all, then, we might justifiably think that Peter's policy was rather sensible: he was concerned for the genuine and deeply felt beliefs f his fellow Jews, and he was not really putting much of an additional demand on the Gentiles. The gospel was not at stake: the Gentile believers had received the
Spirit and been baptized, without circumcision; now all that was required was to accommodate the scruples of the Jewish believers' (S. 482). 
Schäfer (2004:233) betont zu Recht: 'Allein in ihrer eigenen Praxis und für die Dauer ihres Aufenthalts wollen die Jakobusleute nicht von dem Ideal einer möglichst vollständigen Gesetzesbefolgung lassen.'

Ihr Verhalten ist dabei verständlich. Schäfer (2004:233) erinnert daran, dass es dem in Jerusalem gefassten Beschluss 'an einer Regelung betreffs eines "Besuchskontaktes" gesetzesobservanter [...] Jesusjünger in solch gemischten Gemeinden mangelt. ${ }^{56}$ Daher sei der Konflikt nicht innergemeindlichen Ursprungs (weder in Jerusalem noch in Antiochien). Vielmehr zeigt sich in zwischengemeindlichen Kontakten weiterer Klärungsbedarf mit Blick auf das Gemeindeleben. Daher sei die innergemeindliche Dimension des in Antiochien aufgebrochenen Konfliktes von ihrer Entstehung her eher sekundär, auch wenn sie eine ungeheure Sprengkraft entwickelt, wie die folgenden Worte des Paulus zeigen. Paulus wendet sich nicht an die Jakobusleute, sondern an Kephas, dessen Verhaltensänderung eben diese innergemeindliche Dynamik auslöst (nach Schäfer 2004:233).

Diese Verse zeigen, in welchem Ausmaß übergemeindliche Verbindungen - beabsichtigt oder unbeabsichtigt - zu konkretem Verhalten oder Verhaltensänderung geführt (vgl. Schäfer 2004:235-236) und theologische Fragen aufgeworfen, zugespitzt und zu ihrer Klärung beigetragen haben. Dass dies nicht immer konfliktfrei geschah, zeigt die scharfe Reaktion des Paulus. Dass solche Ereignisse noch nach Jahren lebhaft in Erinnerung sein können, zeigt der Bericht im Galaterbrief.

Nach Paulus war die Motivation hinter dem Verhalten des Petrus Furcht: '[W]eil er die aus dem Judentum fürchtete.' Aus der Sicht des Kephas dürfte es sich eher um pragmatisch begründete Rücksicht auf die Gäste gehandelt haben (zu seiner Motivation vgl. Schäfer 2004:236-238). Paulus dürfte die aus Jerusalem angereisten Gäste wohl erst durch ihr Verhalten gleichsam als Eindringlinge empfunden haben, 'die in seinem Verantwortungsbereich das Leben der Gemeinde empfindlich stören' (Schäfer 2004:238). Nach Schäfer (2004) mussten sich die Jakobusleute:

entweder ebenfalls den dortigen Gepflogenheiten anpassen, oder sie sind vermutlich recht bald wieder nach Jerusalem aboder anderswohin gereist, da sie auf ihre Art nicht wirklich in die Gemeinde hineinkommen konnten. (S. 241)

Durch die Reise des Kephas und durch diese Leute entstehen Kontakte zwischen den Gemeinden in Jerusalem und in Antiochien, auch wenn ihr Kommen - beabsichtigt oder unbeabsichtigt - bei wichtigen Gestalten in Antiochien zu einem Verhalten geführt hat, das den heftigen Widerstand des Paulus hervorgerufen hat. Dass übergemeindliche Verbindungen und Besuche auch zu Kontroversen führen

56.Dunn (2009:478-481) spricht von einem "Missing element in the Jerusalem Agreement': 'we have to assume that the issue of Jewish and Gentile believers Agreent' 'we have to assume that the issue of Jewish and Gentile believers eating together had not been raised in the Jerusalem meeting.' Zur Bewertung des Besuchs des Heidenchristen Titus in Jerusalem und der dortigen Tischgemeinschaft durch die Jerusalemer schreibt Dunn (2009): 'As a single God-fearing, or judaizing, Gentile, Titus would have provided no precedent for a whole community (Antioch), probably already consisting of a majority of Gentiles, to ignore what devout Jews regarded as essential' (S. 479). können, zeigt sich im Galaterbrief und anderen Briefen wiederholt auch in der Auseinandersetzung mit den reisenden Gegnern des Paulus. Der Bericht zeigt, dass man mit den Implikationen übergemeindlicher Verbindungen für die Situation vor Ort auf unterschiedliche Weise umgegangen ist.

\section{Galater 4:20; 6:10 und 6:16}

Nach Galater 4:20 ist der vorliegende Brief nur ein dürftiger Ersatz für die leibliche Anwesenheit des Paulus. Wäre er vor Ort, könnte er mit anderer Stimme reden. ${ }^{57}$ Hier wie auch in anderen Briefen (ausgeprägt im 2 Kor) erscheinen Briefe als Kommunikationsmittel, die sich zum einen aus übergemeindlichen Verbindungen ergeben, diese zum anderen aber auch aufrechterhalten und ausbauen. Jedoch verweist die Notiz auch auf die Grenzen, die sich durch schriftliche Kommunikation ergeben: die persönliche Anwesenheit des Apostels würde einen anderen Ton erlauben. Nach Moo (2013) würde Paulus sehr gerne bei den Galatern sein:

$[A]$ nd using the tone of voice appropriate to the friendship that has characterised their previous relations. But the circumstances force him to address them otherwise, using argument and a rebuking tone to bring them back to submission to the 'truth' of the Gospel. (S. 290)

Das Ausmaß der Aufforderung in Galater 6:10, Gutes zu tun an jedermann, 'allermeist aber an des Glaubens Genossen', ist unklar. Bezieht es sich nur auf die Hausgenossen des Glaubens vor Ort oder auch darüber hinaus? In letzterem Fall wären mit der Aufforderung übergemeindliche Implikationen verbunden: Christen an einem Ort wissen von anderen Christen und sind ihnen auch mit Hilfeleistungen verbunden (vgl. oben zu Gal 2:10)..$^{58}$ Dass Paulus solches Engagement von den Galatern erwartet, zeigt sein Versuch, sie später in die Kollektenaktion für Jerusalem einzubinden (1 Kor 16:1: 'wie ich in den Gemeinden in Galatien angeordnet habe, so sollt auch ihr tun'; vgl. Apg 20:4).

In Galater 6:16 verweist Paulus neben den Christen Galatiens hinaus auf eine größere Gruppe von Menschen in den im Brief erwähnten Gebieten und wohl noch darüber hinaus:

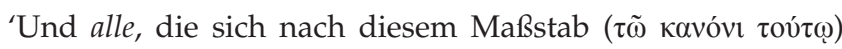
richten - Friede und Barmherzigkeit über sie und über das Israel Gottes.' Die Galater, sofern sie zum Evangelium des Paulus zurückkehren oder sich nicht von ihm abbringen

57.Nach Bruce (1982:213) könnte Paulus dabei befürchten, dass sich die Empfänge auf seine kompromisslose Strenge konzentrieren und die dahinterliegende Fürsorge und Zuneigung übersehen könnten.

58.Bruce (1982) bemerkt, dass damit der Jerusalemer Gemeinde keine Vorrangstellung eingeräumt werde, obwohl sie natürlicherweise auch in den Bereich der Forderung fällt: 'Fellow-Christians had a prior claim on the generosity of their brethren, not only in the same local church but in any or every church. This principle is illustrated in the Jerusalem relief fund, but it is outrunning the evidence to see a specific reference to that fund in this paragraph' (S. 266; kursiv CS), dort ausführliche Diskussion möglicher Bezüge zur Kollektenaktion. Bruce (1982) schreibt: 'These affinities form part of the evidence on which it is argued that these verses (Gal affinities form part of the evidence on which it is argued that these verses (Ga 6:6-10) are specific in intent and form an exhortation to participate in the Jerusalem collection' (L. W. Hurtado, 'The Jerusalem Collection and the Book of Galatians', JSNT 5, 1979:53). If this is so, the allusiveness of the language makes it necessary to suppose that Paul had already communicated with the Galatian about the collection, so that they would understand the present allusion in the light of what they had already been told. Such a prior communication can be neither denied nor affirmed' (S. 266) 
lassen, gehören beziehungsweise sollten zu dieser Gruppe gehören. Dieser Gruppe - und den Galatern, die an diesem Maßstab festhalten oder zu ihm zurückkehren - gilt der Zuspruch von Friede und Barmherzigkeit. Moo resümiert: '[Y]ou Galatians, Paul is saying, must join the unbounded group [...] who will experience God's peace and mercy. This group is composed of those who will follow "this rule"'(Moo 2013:399). ${ }^{59}$

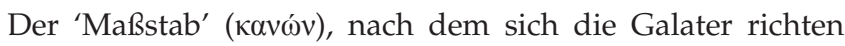
sollen, bezieht sich zunächst auf den unmittelbaren Kontext, den Moo wie folgt beschreibt. Das $\tau$ ov́ $\omega$ impliziere einen Hinweis auf etwas, das Paulus gerade ausgesagt hat. Von daher könnte der Bezug der ganze Vers 15 sein. Aber es ist naheliegender, dass sich der 'Maßstab' auf die 'neue Schöpfung' bezieht, die in Vers 15b erwähnt wird:

Specifically, 'new creation', as Paul has stressed throughout the letter, will prominently feature the work of the Spirit, faith, and the love to which faith gives birth (Gal 5:6) - three key ideas of the letter that otherwise go unmentioned in the letter ending. $\left(\right.$ Moo 2013:399) ${ }^{60}$

Ob sich der кavóv darüber hinaus noch auf eine feste, den Galatern bekannte Größe von Lehrinhalten bezieht, die über die jeweilige Ortsgemeinde hinaus bekannt und Konsens war (vgl. Röm 6:17: 'die Gestalt der Lehre, der ihr ergeben seid', vgl. dazu Stenschke 2017:256), ist unsicher. Falls die

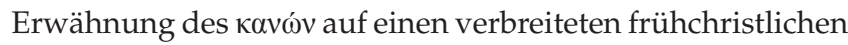
Lehrkonsens beziehungsweise Katechismus hinweist, würde auch dies für übergemeindliche Verbindungen sprechen. Dies wurde von Alfred Seeberg vertreten ([1903] 1966), der annahm, dass diese Lehre auch ethische Vorgaben enthielt (vgl. Eph 4:1; Phil 1:27; Kol 1:10; 1 Thess 2.12). ${ }^{61}$ Die Verbreitung einer solchen Zusammenfassung christlicher Lehre würde nicht nur erhebliche übergemeindliche Verbindungen voraussetzen, sondern auch das Bemühen, gemeindeübergreifende und Einheit stiftende Lehrstücke zu formulieren, bezeugen.

\section{Zusammenfassung und Implikationen}

Im Galaterbrief erscheinen mehrere direkte und indirekte Hinweise auf übergemeindliche Verbindungen. Wie in anderen Briefen des Apostels, liegt der Fokus auf Verbindungen, die durch Paulus zustande gekommen waren und gehalten wurden. Über die Rolle und Motivation anderer

\section{7 ur Diskussion, wie sich die Gruppe derer, die sich an diesen Maß̌stab halten zur abschließend erwähnten Größe 'das Israel Gottes' verhalten, vergleiche Moo (2013) Seiten 401-403. Wenn mit dem 'Israel Gottes' nicht die Gruppe derer, die sich an diesen Maßstab halten, gemeint ist (also die christliche Gemeinde, so Moo mit Begründung), sondern das ethnische Israel, dann würden die Galater zu eine Größe gehören, die ähnlich wie das Israel Gottes an mehreren Orten lebt und au verschiedene Weise überörtlich miteinander verbunden ist; vergleiche die Diskussion der Bedeutung dieses Ausdrucks bei Barclay (2015;420-421): '[T]he nation of Israel [...] On this reading, it would also be mistaken to claim that (unbelieving) Israel lies beyond the horizon of this letter'; vergleiche auch Bachmann (1999;159-189, 2010).}

60.Ähnlich Bruce (1982): 'The kavẃv is apparently the principle just laid down about the new creation': the reference then is to members of the new humanity who are guided by this principle, in contrast to those who maintain the continuing validity of circumcision and similar legal requirements' (S. 273).

61.Nach Stuhlmacher (1998:89) ist schon das 'Evangelium' in Römer 1:16-17 von Galater 6:17 her zu verstehen.
Christen (Kephas, Jakobus, die Jakobus-Gruppe, Barnabas und andere) lässt sich - aufgrund der knappen Angaben des Paulus - nur mutmaßen. ${ }^{62}$

Paulus misst diesen Verbindungen an Schlüsselstellen seiner Argumentation große Bedeutung zu: seine Verbindungen zu anderen Christen beziehungsweise seine Unabhängigkeit von ihnen sind entscheidend. Die Hinweise in Galater 1-2 erscheinen in einem Kontext, in dem Paulus die Unabhängigkeit seiner Berufung und seines Evangeliums betont; in einem anderen argumentativen Zusammenhang trüge die Darstellung eventuell andere Akzente.

Zusammen mit anderen Christen schreibt Paulus den Brief an mehrere Gemeinden (Gal 1:1-2). Neben Paulus und seinen Mitarbeitern entstehen Verbindungen auch durch andere 'Christen', gegen die Paulus freilich energisch vorgeht, weil sie ein anderes Evangelium verkündigen (Gal 1:7; 4:17; 5:78.10.12; 6:12). Thre übergemeindlichen Verbindungen dienen wohl dazu, die Situation ihrer Heimatgemeinden zu entschärfen. Die Praxis paulinischer Mission ist über christliche Gemeinden hinaus bekannt geworden. Paulus widerlegt detailliert Gerüchte und Anschuldigungen, die gegen ihn unter den Gemeinden im Umlauf sind. Nachrichten über Paulus sind unter den Gemeinden größerer Gebiete bekannt (Gal 1:13). Durch die Missionsarbeit des Paulus (und etwaiger Mitarbeiter) entstehen Verbindungen zwischen bestehenden (Damaskus, Jerusalem) und neu gegründeten Gemeinden in Arabien, Syrien und Kilikien (Gal 1:21). Die Kunde von der Lebenswende des Paulus findet dank entsprechender Verbindungen weite Verbreitung (Gal 1:23).

Eine entscheidende Rolle in der Argumentation spielen vier Besuche, die übergemeindliche Verbindungen ausdrücken und ihre zum Teil weitreichende Bedeutung illustrieren. Bei zwei Besuchen geht die Initiative von Paulus aus: Bei seinem ersten Besuch kommt er von sich aus nach Jerusalem, um Kephas kennenzulernen, ferner trifft er Jakobus (Gal 1:1819). Trotz eventueller Implikationen für seinen Dienst war dieser Besuch eher privater Natur, auch wenn die Beteiligten Mitglieder und Repräsentanten von Gemeinden sind. Bei seinem zweiten Besuch kommt Paulus aufgrund einer Offenbarung in Begleitung anderer (Barnabas und Titus) und wohl im Auftrag von Gemeinden, um seine Verkündigung mit den Jerusalemer 'Säulen' Jakobus, Kephas und Johannes abzugleichen, Anerkennung für seine gesetzesfreie Mission unter Heiden ('die Gnade, die mir gegeben war') zu finden, um nicht vergebens $\mathrm{zu}$ arbeiten (an Titus demonstriert) und um Arbeitsbereiche abzustecken. Beide Besuche hatten Implikationen für die von Paulus gegründeten Gemeinden ('damit die Wahrheit des Evangeliums bei euch bestehen bliebe').

Zwei weitere Besuche gehen von anderen Judenchristen aus und sind potentiell mit weitreichenden Entscheidungen

62. Weitere Schlüsse sind möglich, wenn man die Apostelgeschichte heranzieht. Jedoch ist die Zuordnung der erwähnten Ereignisse, vor allem Galater 2:1-10, zur Jedoch ist die Zuordnung der erwähnten Ereignisse, vor allem Galater 2:1-10, zur
Schilderung der Apostelgeschichte umstritten; vergleiche Wenham (1993) und Zeigan (2005). 
verbunden (Gal 2:11-14). Kephas kommt aus nicht genannten Gründen nach Antiochien und passt sich zunächst der Praxis der antiochenischen Gemeinde an. Das Kommen der Jakobus-Gruppe zeigt übergemeindliche Verbindungen über die führenden Persönlichkeiten hinaus. Die Reise und Ankunft dieser Gruppe in Antiochien an sich ist nicht umstritten. Ihr Kommen löst bei Kephas, Barnabas und anderen Judenchristen konkrete Verhaltensänderungen aus. Umstritten ist die Rücksichtnahme der antiochenischen Judenchristen und die Aussetzung der Tischgemeinschaft mit den Heidenchristen. Dies führt seitens des Paulus zu heftiger Diskussion und Klärung des Wesens des Evangeliums und judenchristlicher Praxis. Hier zeigt sich, dass die Folgen übergemeindlicher Verbindungen vor Ort wichtige theologische Fragen aufwerfen konnten und $\mathrm{zu}$ ihrer Klärung beigetragen haben (hier: wie sich Judenchristen in Gemeinden aus Judenchristen und Heidenchristen verhalten sollen, evtl. noch grundsätzlicher: Bedeutung und Geltung des Gesetzes für Judenchristen; Wesen des christlichen Evangeliums). Eindrucksvoll schildert Schäfer das Dilemma, in dem sich Petrus und eventuell auch andere Judenchristen in Antiochien nach Ankunft der JakobusGruppe befanden. Der in Jerusalem erreichte Konsens bezüglich der Heidenchristen (Gal 2:1-10) hat Implikationen in einem wohl mehrheitlich heidenchristlichen Kontext. Das ging - zumindest in der Perspektive des Paulus - nicht ohne grundsätzliche Klärung der Bedeutung des Gesetzes und der Soteriologie. Daher haben manche übergemeindlichen Verbindungen und ihre Folgen vor Ort eine wichtige Bedeutung für die Entwicklung urchristlicher Theologie und Praxis.

Dabei zeigt sich auch, dass, auch wenn übergemeindliche Verbindungen gleichsam als 'Katalysator' wirken können, sie nicht notwendigerweise oder von selbst $\mathrm{zu}$ einer einheitlichen Theologie und Praxis führen oder die theologische Einheit des Neuen Testaments a priori gewährleisten: die Jakobus-Gruppe hat sich der antiochenischen Praxis der Tischgemeinschaft von Judenund Heidenchristen nicht angepasst, eventuell ist sie auch der paulinischen Position bezüglich der Bedeutung des Gesetzes nicht gefolgt. Zumindest zu einigen Jerusalemern blieb sein Verhältnis angespannt. Die hier relativ detailliert geschilderten übergemeindlichen Verbindungen durch wechselseitige Besuche und ihre Implikationen bieten einen Einblick in das 'Labor' urchristlicher Theologie.

Paulus schreibt den Galatern ins Stammbuch, dass es beim Festhalten an seinem Evangelium um nichts weniger als um Frieden und Barmherzigkeit geht (Gal 6:16). Nur wer diesem 'Kanon' folgt, gehört zum größeren Ganzen des Volkes Gottes. Andere Hinweise auf übergemeindliche Verbindungen sind unsicher (Gal 2:10, 6:10).

Diese Ergebnisse haben Implikationen für die eingangs erwähnten Fragestellungen:

1. Die Hinweise im Galaterbrief zeigen, dass und in welchem Ausmaß übergemeindliche Verbindungen im Urchristentum tatsächlich vorhanden waren beziehungsweise zumindest vorausgesetzt werden. Sie sind keineswegs auf Paulus begrenzt. Solche Verbindungen werden in Vergleichen mit antiken Vereinen also nicht pauschal konstatiert, ${ }^{63}$ sondern lassen sich im Detail nachweisen. Die Anfragen Ascoughs (1997) an die tatsächlich vorhandenen urchristlichen 'translocal links' können damit als widerlegt gelten. ${ }^{64}$

2. Neben den 'großen Gestalten', die nach wie vor die Geschichtsschreibung des Urchristentums dominieren, werden die im Galaterbrief vorhandenen übergemeindlichen Verbindungen (vor allem Gal 2:1-14) zu Recht bei der Untersuchung und Darstellung der Geschichte des Urchristentums gebührend berücksichtigt. Angesichts der Vorkommen im Galaterbrief ist $\mathrm{zu}$ begrüßen, dass Barnabas und Titus in der neueren Forschung stärker beachtet werden. Sie und andere Mitarbeiter spielen bei den übergemeindlichen Verbindungen eine wichtige Rolle. Es fehlt eine PaulusMonografie, die umfassend das Wirken der Mitarbeiter als Mitverfasser und eigenständig agierende Missionare und Theologen sowie die Einbindung des Paulus in das größere Netzwerk urchristlicher Gemeinden umfassend berücksichtigt. ${ }^{65}$ Damit würde allerdings die akademische Gattung 'Paulus-Buch' verlassen und eine Geschichte des paulinischen Christentums beziehungsweise der paulinischen Mission entstehen. Ferner liegen mehrere neuere Studien zu den Gegnern des Paulus vor, die neben den übergemeindlichen Verbindungen im Sinne des Paulus weitere urchristliche Netzwerke bezeugen.

3. Die im Galaterbrief erwähnten übergemeindlichen Verbindungen unterstützen die These Bauckhams hinsichtlich der weiteren Adressatenschaft der Evangelien. Im einführenden Aufsatz des Sammelbandes The Gospels for all Christians, schreibt Bauckham (1998):

The early Christian movement was not a scattering of isolated, self-sufficient communities with little or no communication between them, but quite the opposite: a network of communities with constant, close communication among themselves. In other words, the social character of early Christianity was such that the idea of writing a Gospel purely for one's own community is unlikely to have occurred to anyone. The consensus I am challenging seems to depend on a view of an early Christian community as a self-contained, self-sufficient, introverted group, having little contact with other Christian communities and little sense of participation in a worldwide Christian movement. Identity, issues, and concerns, it seems to be presupposed, are thoroughly local. (S. 25)

63.Etwa Meeks (1983:80): "[T]he associations offer as little help as the household in explaining the extralocal linkages of the Christian movement.'

64.Neben der knappen Kritik Schmellers (2001) müssten umfangreiche Studien zeigen, ob und in welchem Ausmaß es tatsächlich überörtliche Verbindungen zwischen antiken Vereinen gab.

65.Ansätze bei Horn (2013) und Schnelle (2014). Schnelle betont, dass sich die Arbeit des Paulus innerhalb des großen Mitarbeiterkreises kaum auf reine Organisationsfragen beschränkt haben dürfte. Er schreibt:'Die Vorstellung eines monolithischen Paulus, der einsam und heroisch seine Theologie entwickelt und weitreichende Entscheidungen trifft, ist nicht nur lebensfremd, sondern wird durch
den Briefbefund widerlegt. Die ouvepyoi [...] waren nicht von Paulus Beauftragte, sondern von Gott in Dienst Genommene (vgl. 1 Kor 3:9). Wie Paulus arbeiten auch (vich (vgl. 1 Thess 3:2; 1 Kor 3:5-9; 16:10.15-18; 2 Kor 8:16-23; Phil 2:22). Speziell im engeren Mitarbeiterkreis wird man deshalb eine intensive theologische Arbeit voraussetzen dürfen' (S. 144). 
4. Dieser rege Austausch betrifft auch die Frage nach dem Verhältnis von theologischer Einheit und Vielfalt im Neuen Testament. Was für die Leserschaft der Evangelien gilt, hat auch Implikationen für die Entwicklung urchristlicher Theologie. Auch hier sollte man nicht vorschnell unterschiedliche Entwicklungen und Akzente mit Isolation und Abgrenzung erklären oder von solchen Konstruktionen her konstatieren. Angesichts der vorhandenen, vielfältigen übergemeindlichen Verbindungen und dem damit gegebenen Austausch an Personen und Informationen aller Art, ist die Vorstellung völlig disparater theologischer Entwicklungen im Urchristentum wenig plausibel. Eher ist mit örtlichen Akzentuierungen eines gemeindeübergreifenden Konsenses zu rechnen. In der neueren Diskussion um die Einheit und Vielfalt neutestamentlicher Theologie wären übergemeindliche Verbindungen als materielle Grundlage und Ermöglichung theologischer Einheit stärker zu berücksichtigen. So wie theologische Einheit nicht nur konstatiert, sondern tatsächlichnachgewiesen werden kann (u.a. das Anliegen und der Verdienst von Hahn 2002:1-36, 799-806), lässt sich diese Einheit - zumindest auch - mit den vorhandenen übergemeindlichen Verbindungen erklären. Die übergemeindlichen Verbindungen des Galaterbriefs zeigen zum einen, dass um die theologische Einheit gerungen und Übereinstimmung und Anerkennung angestrebt wurde (Besuche des Paulus in Jerusalem). Zumindest in Einzelfällen dienten übergemeindliche Verbindungen und die mit ihnen aufgeworfenen Fragen bezüglich Lehre und Praxis als Katalysatoren für die Entwicklung urchristlicher Theologie. Zum anderen wird deutlich, dass übergemeindliche Verbindungen nicht notwendig zu einheitlichen Entwicklungen führen. Kephas und die Jakobus-Gruppe haben sich bei Kontakten mit anderen Christen unterschiedlich verhalten. $\mathrm{Ob}$ sie $\mathrm{zu}$ einer Reflektion ihrer Position und Verhaltensänderung bereit waren, wird nicht erwähnt.

Ferner sind übergemeindliche Verbindungen bei der Beschreibung der neutestamentlichen Ekklesiologie zu berücksichtigen. Sie zeigen, dass einzelne Gemeinden kein isoliertes Phänomen sind, sondern zum größeren Ganzen der universalen Kirche gehören. Freilich bleibt es schwer einzuschätzen, inwieweit dieses 'größere Ganze' mehr oder etwas entscheidend anderes ist als die jeweiligen einzelnen Gemeinden oder die Summe der Gemeinden. Ein größeres Ganzes zu dem die einzelnen Gemeinden gehören, ist jedenfalls keine nur abstrakte theologische Vorstellung, etwa des Epheserbriefs, sondern auch in vielfältigen Verbindungen erkennbar.

5. Die hier zusammengetragenen Hinweise bieten wichtige Rohdaten für eine noch ausstehende, umfassende soziale Netzwerkanalyse der paulinischen Mission und Gemeinden. ${ }^{66}$ Mit dem Hinweis auf 'alle' Christen in Galater 6:16 geht das Ausmaß dieses Netzwerks jedoch noch darüber hinaus.

66.Die Studie von Rollinger (2014) zeigt, dass die Aufbereitung dieser Angaben zu Datensätzen, die für eine computergestützte Analyse brauchbar sind, ein komplexes Unterfangen ist. Der Reiz richtig erstellter Datensätze liegt darin, dass komplexes Unterfangen ist. Der Reiz richtig erstellter Datensätze liegt darin, dass
man mehrere Fragestellungen mit unterschiedlichen Parametern und Ergebnissen über sie laufen lassen kann.

\section{Bestätigungen Konkurrierendes Interesse}

Der Autor erklärt offiziell, dass er keine finanziellen oder persönlichen Beziehungen hatten, die ihn beim Schreiben dieses Artikels möglicherweise unangemessen hätte beeinflussen können.

\section{Literaturverzeichnis}

Arterbury, A.E., 2005, Entertaining angels: Early Christian hospitality in its Mediterranean setting, Sheffield Phoenix Press, Sheffield. (New Testament Monographs)

Ascough, R.S., 1997, 'Translocal relationships among voluntary associations and early Christianity', Journal of Early Christian Studies 5, 223-241.

Bachmann, M., 1999, 'Kirche und Israel Gottes', in M. Bachmann (ed.), Antijudaismus im Galaterbrief? Exegetische Studien zu einem polemischen Schreiben und zur Theologie des Apostels Paulus, pp. 159-189, Vandenhoeck \& Ruprecht, Göttingen. (Novum Testamentum et orbis antiquus, 40)

Bachmann, M., 2010, 'Bemerkungen zur Auslegung zweier Genetivverbindungen des Galaterbriefs: "Werke des Gesetzes" (Gal 2:16 u.ö.) und "Israel Gottes" (Gal 6:16)', in ders. und B. Kollmann (Hrsg.), Umstrittener Galaterbrief: Studien zur Situierung und Theologie des Paulus-Schreibens, S. 95-118, Neukirchener, Neukirchen-Vluyn. (Biblisch-theologische Studien, 106)

Barclay, J.M.G., 2015. Paul and the gift, Eerdmans, Grand Rapids, MI.

Bauckham, R.I., 1998, 'For whom were the Gospels written?', in R. Bauckham (ed.) The Gospels for all Christians: Rethinking the Gospel audiences, pp. 9-48, T. \& T. Clark, Edinburgh.

Best, E., 1988, Paul and his converts, T. \& T. Clark, Edinburgh.

Bruce, F.F., 1982, The Epistle to the Galatians: A commentary on the Greek text, Eerdmans, Exeter. (New International Greek Text Commentary)

Campbell, D.A., 2014, Framing Paul: An epistolary biography, Eerdmans, Grand Rapids, Ml.

Deines, R., 2017, Jakobus: Im Schatten des Größeren, Evangelische Verlagsanstalt, Leipzig. (Biblische Gestalten, 30)

Dickson, J.P., 2003, Mission-commitment in ancient Judaism and in the Pauline communities: The shape, extent and background of early Christian mission, Mohr Siebeck, Tübingen. (Wissenschaftliche Untersuchungen zum Neuen Testament, II.159)

Driedger, A., 2015, 'Der sogenannte "antiochenische Zwischenfall" (Gal 2:11-14) und seine Bedeutung für die Geschichte und Theologie des Urchristentums', MTh-Dissertation, University of South Africa, Pretoria.

Dunn, J.D.G., 2009, Beginning from Jerusalem: Christianity in the making, II, Eerdmans, Grand Rapids, MI.

Hahn, F., 2002, Theologie des Neuen Testaments, II, Die Einheit des Neuen Testaments. Thematische Darstellung, Mohr Siebeck, Tübingen.

Hansen, G.W., 1993, s.v. 'Rhetorical criticism', in G.F Hawthorne, R.P. Martin \& D.G. Reid (eds), Dictionary of Paul and his letters: A compendium of contemporary scholarship, InterVarsity Press, Downers Grove, pp. 822-826.

Hengel, M. \& Schwemer, A.M., 1997, Paul between Damascus and Antioch: The unknown years, SCM, London.

Hezser, C., 2011, Jewish travel in antiquity, Mohr Siebeck, Tübingen. (Texts and Studies in Ancient Judaism, 144)

Horn, F.W. (ed.), 2013, Paulus Handbuch, Mohr Siebeck, Tübingen. (TheologenHandbücher)

Jewett, R., 1970-1971, 'The agitators and the Galatian congregation', New Testament Studies 17, 198-121.

Kollmann, B., 1998, Joseph Barnabas Leben und Wirkungsgeschichte, Katholisches Bibelwerk, Stuttgart. (Stuttgarter Bibelstudien, 175)

Meeks, W.A., 1983, The first urban Christians: The social world of the Apostle Paul, Yale University Press, New Haven.

Moo, D.J., 2013, Galatians, Baker, Grand Rapids, MI. (Baker Exegetical Commentary on the New Testament)

Nanos, M.D., 2005, 'Intruding, "Spies" and "Pseudo-brethren": The Jewish intra-group politics of Paul's Jerusalem meeting', in S.E. Porter (ed.), Paul and his opponents, pp. 59-97, Brill, Leiden. (Pauline Studies, 2)

Öhler, M., 2002, Barnabas, Mohr Siebeck, Tübingen. (Wissenschaftliche Untersuchungen zum Neuen Testament, 156).

Öhler, M., 2013, 'Mitarbeiter und Mitarbeiterinnen des Paulus', in F.W. Horn (Hrsg.), Paulus Handbuch, S. 243-256, Mohr Siebeck, Tübingen. (Theologen-Handbücher)

Ollrog, W.-H., 1979, Paulus und seine Mitarbeiter: Untersuchungen zur Theorie und Praxis der paulinischen Mission, Neukirchener, Neukirchen-Vluyn. (Wissenschaftliche Monografien zum Alten Testament, 50).

Park, Y.-H., 2015, Paul's Ekklesia as civic assembly: Understanding the people of God in their politico-social world, Mohr Siebeck, Tübingen. (Wissenschaftliche Untersuchungen zum Neuen Testament, II.393). 
Reicke, B., 1982, Neutestamentliche Zeitgeschichte: Die biblische Welt von 500 v. Chr. bis 100 n. Chr., 3. Aufl., W. de Gruyter, Berlin, NY. (De Gruyter Lehrbuch).

Riesner, R., 1998, Paul's early period: Chronology, mission strategy, theology, Eerdmans, Grand Rapids, MI.

Rollinger, C., 2014, Amicitia sanctissime colenda: Freundschaft und soziale Netzwerke in der späten Republik, Verlag Antike, Heidelberg. (Studien zur Alten Geschichte, 19).

Schäfer, R., 2004, Paulus bis zum Apostelkonzil: Ein Beitrag zur Einleitung in den Galaterbrief, zur Geschichte der Jesus-Bewegung und zur Pauluschronologie, Mohr Siebeck, Tübingen. (Wissenschaftliche Untersuchungen zum Neuen Testament, II.179).

Schmeller, T., 2001, 'Neutestamentliches Gruppenethos', in J. Beutler (Hrsg.), Der neue Mensch in Christus: Hellenistische Anthropologie und Ethik im Neuen Testament, S. 120-143, Herder, Freiburg. (Questiones Disputatae 190)

Schnabel, E.J., 2008, Paul, the missionary: Realities, strategies and methods, IVP/ Apollos, Nottingham.

Schnelle, U., 2014, Paulus: Leben und Denken, 2. Aufl., De Gruyter, Berlin,. (De Gruyter Studium).

Schweizer, E., 1989, Der Brief an die Kolosser, 3. Aufl., Benziger, Zürich. (EvangelischKatholischer Kommentar zum Neuen Testament, 12)

Seeberg, A., [1903] 1966, Der Katechismus der Urchristenheit, Ch. Kaiser, München (Theologische Bücherei Neues Testament, 26)

Siebenthal, H.von, 2011, Griechische Grammatik zum Neuen Testament, Brunnen, Gießen.

Stenschke, C.W., 2011, 'Sandten die Apostel zu ihnen Petrus und Johannes' (Apg 8:10): Überörtliche Verbindungen der urchristlichen Gemeinden in der Darstellung der Apostelgeschichte des Lukas', Ephemerides Theologicae Lovanienses 87, 433-453.

Stenschke, C.W., 2017, 'Übergemeindliche Verbindungen und ihre Funktion im Römerbrief', Estudios Bíblicos 75, 245-281.

Stenschke, C.W., 2018, "'Samt allen, die den Namen unseres Herrn Jesus Christus anrufen an jedem Ort, bei innen und bei uns" (1 Kor 1:2): Übergemeindliche Verbindungen und ihre Funktion im 1 Korintherbrief', Estudios Biblicos 76(2), 265-297.
Stenschke, C.W., 2018, "Samt allen Heiligen in ganz Achaia" (2 Kor 1:1): Übergemeindliche Verbindungen im Brief des Paulus und Timotheus an die Gemeinden Korinths und Achaias', Ephemerides Theologicae Lovanienses 94(3), 601-634.

Stuhlmacher, P., 1998, Der Brief an die Römer, 2. Aufl., Vandenhoeck \& Ruprecht, Göttingen. (Neues Testament Deutsch, 6 )

Sumney, J.L., 1999, 'Servants of Satan', 'False Brothers' and other opponents of Paul, Sheffield Academic Press, Sheffield. (Journal for the Study of the New Testament Supplement Series, 188)

Sumney, J.L., 2005, 'Studying Paul's opponents: Advances and challenges', in S.E. Porter (ed.), Paul and his opponents, pp. 7-58, Brill, Leiden. (Pauline Studies, 2)

Taylor, N.H., 2005, 'Apostolic identity and the conflicts in Corinth and Galatia', in S.E. Porter (ed.), Paul and his opponents, pp. 99-123, Brill, Leiden. (Pauline Studies, 2)

Thompson, M.B., 1998, 'The holy internet: Communications between churches in the first Christian generation', in R. Bauckham (ed.), The gospels for all Christians: Rethinking the gospel audiences, pp. 49-70, T. \& T. Clark, Edinburgh.

Von Lips, H., 2016, Timotheus und Titus. Unterwegs für Paulus, 3. Aufl., Evangelische Verlagsanstalt, Leipzig. (Biblische Gestalten, 19)

Von Siebenthal, H., 2011, Griechische Grammatik zum Neuen Testament, Brunnen, Basel.

Wenham, D., 1993, 'Acts and the Pauline Corpus: II., The evidence of parallels', in B.W. Winter \& A.D. Clarke (eds.), Ancient literary setting, pp. 215-258, Eerdmans, Grand Rapids. (The Book of Acts in its first Century setting, 1)

Williams, M.H., 2015, 'From Shimon to Petros - Petrine nomenclature in the light of contemporary onomastic practices', in H.K. Bond \& L.W. Hurtado (eds.), Peter in early Christianity, pp. 30-45, Eerdmans, Grand Rapids, MI.

Zeigan, H., 2005, Aposteltreffen in Jerusalem: Eine forschungsgeschichtliche Studie zu Gal 2,1-10 und den möglichen lukanischen Parallelen, Evangelische Verlagsanstalt, Leipzig. (Arbeiten zur Bibel und ihrer Geschichte, 18) 